\title{
New cyclopentadienylethylphosphane chelate complexes with unsymmetrical phosphane substitution $\dagger \ddagger$
}

\author{
Karin Janssen (née Kirleis) and Holger Butenschön* \\ Received (in Victoria, Australia) 3rd April 2011, Accepted 13th June 2011 \\ DOI: $10.1039 / \mathrm{c} 1 \mathrm{nj} 20292 \mathrm{~h}$
}

The syntheses, characterization, and some reactions of (phosphanylethyl)cyclopentadienyl chelate complexes of cobalt, rhodium, iridium, nickel, and chromium with unsymmetrical substitution at the phosphorus atom are described. The ligand systems were prepared by nucleophilic ring opening of spiro[2.4]hepta-4,6-diene with lithium tert-butylphenylphosphide or lithium tert-butylcyclohexylphosphide. The anionic ligands give the respective chelate complexes by treatment with metal halide reagents. In three cases it was possible to obtain X-ray crystal structure analyses. The cobalt chelate complex undergoes oxidative addition with a dihydrosilane, the reaction results in the formation of products with three stereogenic centers at phosphorus, cobalt, and silicon, which show dynamic behavior as indicated by VTNMR.

The rhodium chelate complex undergoes oxidative addition of iodomethane with diastereoselective formation of the respective $\mathrm{Rh}$ (III) chelate. While diastereoselectivity caused by a planar chiral indenyl ligand or by a stereogenic carbon center in the chelate backbone has earlier been observed, this is the first case of a stereoinduction by the stereogenic phosphorus ligand. Activation energies for the rotation of cobalt and rhodium chelates have also been determined by VTNMR.

\section{Introduction}

Cyclopentadienyl $(\mathrm{Cp})$ complexes are among the most common ones in organometallic chemistry and have found various applications in fields such as catalysis, ${ }^{1}$ synthesis, ${ }^{2}$ materials sciences, ${ }^{3,4}$ medicinal chemistry ${ }^{3-6}$ and many more. ${ }^{7-11}$ One characteristic feature of cyclopentadienyl ligands is their facile rotation around the axis between the center of the Cp ligand and the metal atom. ${ }^{12}$ Such a rotation is impossible in $\mathrm{Cp}$ chelate complexes, in which the $\mathrm{Cp}$ ligand is connected to other coordinated moieties. The chemistry of $\mathrm{Cp}$ complexes with heteroatomic tethers, e.g. phosphane complexes such as $\mathbf{1}$ has been reviewed some time ago, and complexes with an ethylene tether $(n=1)$ are the most prominent representatives of this class of chelates. ${ }^{13,14}$ Most complexes of this kind have two identical substituents at the phosphorus atom. This includes a number of cobalt and nickel complexes reported by our group, which have a di-tert-butyl substitution pattern, such as complexes $\mathbf{2}$ and 3 . $^{13,15-30}$

Institut für Organische Chemie, Leibniz Universität Hannover,

Schneiderberg 1B, D-30167 Hannover, Germany.

E-mail: holger.butenschoen@mbox.oci.uni-hannover.de

$\dagger$ Dedicated to Didier Astruc on the occasion of his 65th birthday.

$\ddagger$ CCDC reference numbers 820401-820403. For crystallographic data

in CIF or other electronic format see DOI: $10.1039 / \mathrm{c} 1 \mathrm{nj} 20292 \mathrm{~h}$

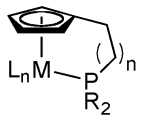

1

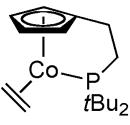

2

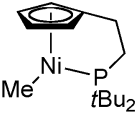

3
Complexes with two different substituents at phosphorus are comparatively rare. The few, in some cases rather special examples of such complexes, have been reported by Nakazawa and Miyoshi et al. (Zr, Hf), ${ }^{31-33}$ Hey-Hawkins et al. $(\mathrm{Ti}, \mathrm{Zr}),{ }^{34}$ Ganter et al. (Mn, Ru), ${ }^{35-37}$ and Saunders et al. (Rh) ${ }^{38-41}$ Different substituents at phosphorus render the complexes less symmetric and are expected to affect the chemical and spectroscopic properties of the complexes as well as the energy of activation of an ethene rotation, which has earlier been reported for $2 .{ }^{27}$ In order to get a deeper insight into these aspects we prepared the first cyclopentadienylethylphosphane chelates of cobalt, rhodium, iridium, nickel, and chromium with tert-butyl and phenyl or with tert-butyl and cyclohexyl substituents at phosphorus. Three of these complexes were characterized by crystal structure analyses.

\section{Results and discussion}

As first shown by Kauffmann et al., spiro[2.4]hepta-4,6-diene (4) is the superior starting material for the synthesis of cyclopentadienylethylphosphane ligands. ${ }^{42}$ The ligands, which 
are obtained by treatment of $\mathbf{4}$ with the respective phosphide, are treated with metal halides to form the respective cyclopentadienyl complexes.

Reaction of $\mathbf{4}$ with lithium tert-butylphenylphosphide followed by addition of $\mathrm{CoCl}_{2}$, chromatographic product isolation and recrystallization from tert-butyl methyl ether (TBME)/petroleum ether (PE) at $-25^{\circ} \mathrm{C}$ gave paramagnetic chloro chelate $\mathrm{rac}-\mathbf{5}$ as purple crystals in $65 \%$ yield. rac-5 was characterized spectroscopically by the IR and MS data obtained in addition to an elemental analysis. The data obtained resemble those of the closely related di-tert-butyl substituted complex. ${ }^{28,29}$ Subsequent treatment of $\mathrm{rac}-\mathbf{5}$ with sodium amalgam $(1 \%)$ and ethene in THF at $-78{ }^{\circ} \mathrm{C}$ followed by warming to $25^{\circ} \mathrm{C}$ gave ethene chelate complex rac-6 in $40 \%$ yield.
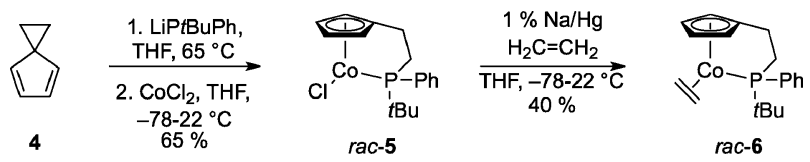

As a consequence of the asymmetric substitution pattern at phosphorus the ${ }^{1} \mathrm{H}$ NMR spectrum of rac-6 is more complicated than that of 2 . The cyclopentadienyl protons give rise to a ABCD line system, and the diastereotopic methylene protons 6-H give rise to two signals, which appear as multiplets. The ethene ligand shows three clearly separated ${ }^{1} \mathrm{H}$ NMR signals at $\delta=2.05(\mathrm{~m}, 2 \mathrm{H}), 2.44(\mathrm{~m}, 1 \mathrm{H})$, and $2.73(\mathrm{~m}, 1 \mathrm{H}) \mathrm{ppm}$, whereas the corresponding signals of $\mathbf{2}$ are observed at $\delta=1.87(2 \mathrm{H})$ and $2.27(\mathrm{~m}, 2 \mathrm{H}) \mathrm{ppm}$. This clearly attests for the asymmetry of the compound and for the profound difference of the electronic environment of the ethene protons as a result of the magnetic anisotropy of the phenyl substituent. Remarkably, a corresponding difference is not observed for the ${ }^{13} \mathrm{C} N M R$ signal assigned to the ethene carbon atoms, which give rise to only one signal at $\delta=22.9 \mathrm{ppm}$, a value very close to the corresponding one observed for $2(\delta=22.4 \mathrm{ppm}) .{ }^{27}$ Dynamic ${ }^{1} \mathrm{H}$ NMR measurements allowed for the determination of the activation energy of the hindered rotation of the ethene ligand around the ethene-cobalt axis in $\mathbf{2}$, which was found to be $62 \mathrm{~kJ} \mathrm{~mol}^{-1} \cdot{ }^{29}$ For 6 the respective coalescence was observed at $350 \mathrm{~K}(400 \mathrm{MHz})$ corresponding to an estimated activation energy of $68.7 \mathrm{~kJ} \mathrm{~mol}^{-1}$. ${ }^{43}$ This value is somewhat higher than that obtained for 2, $\mathrm{CpNi}\left(\mathrm{C}_{2} \mathrm{H}_{4}\right) \mathrm{CH}_{3}$, and $\mathrm{CpRh}\left(\mathrm{C}_{2} \mathrm{H}_{4}\right)_{2}$, but significantly lower than that for $\mathrm{CpCo}\left(\mathrm{C}_{2} \mathrm{H}_{4}\right)_{2} \cdot{ }^{43,44}$

Crystallization from hexane at $-25{ }^{\circ} \mathrm{C}$ afforded crystals, which were suitable for an X-ray crystal structure analysis (Fig. 1). The structure shows a torsion of the ethylene bridge, which is typical for this class of complexes and allows a minimization of $\mathrm{H}-\mathrm{H}$ interactions in the bridge. Remarkably, the cyclopentadienyl ring and the phenyl substituent adopt an almost coplanar orientation. In contrast to the NMR investigation the asymmetry of the phosphane substitution is not structurally reflected in the ethene ligand, both carbon atoms show almost identical distances to the cobalt atom.

Chelate 2 has been shown to undergo oxidative addition reactions with hydrosilanes resulting in hydridosilylcobalt(III) chelates. ${ }^{18}$ When a dihydrosilane was used the process generates a new stereogenic center at silicon as well as at cobalt.

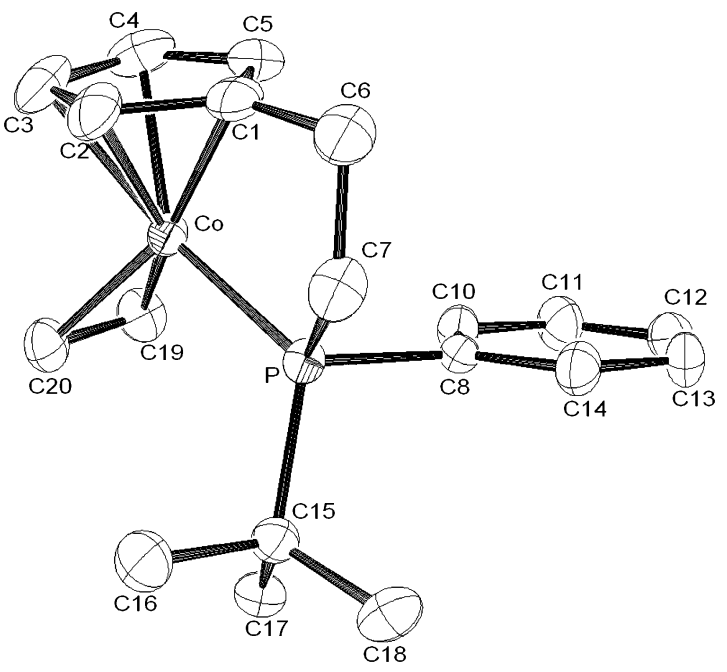

Fig. 1 Structure of rac-6 in the crystal. ${ }^{45}$ Displacement ellipsoids correspond to the $50 \%$ probability level. Selected bond lengths [pm] and angles [ ${ }^{\circ}$ ]: Co1-P1 215.76(9), Co1-C1 205.2(3), Co1-C2 208.3(3), Co1-C3 207.4(3), Co1-C4 209.1(3), Col-C5 208.8(3), Co1-C19 200.8(3), Co1-C20 200.8(3), C1-C6 150.8(4), C6-C7 152.7(4); C1-C6-C7 109.9(2), C6-C7-P1 105.43(19), Co1-P1-C7 103.08(10).

However, the diastereomeric excess was only $23 \%$ in the reported case. In contrast to $\mathbf{2}$ chelate rac-6 has an additional stereocenter at the phosphorus atom. Consequently, the respective reaction of $r a c-6$ with methylphenylsilane was expected to give four diastereomers. The experiment showed that this was indeed the case, addition of methylphenylsilane to $r a c-6$ afforded complex rac-7 in $71 \%$ yield as a mixture of diastereomers in the ratio $1.00: 0.95: 0.56: 0.48$ as determined by deconvolution calculations from the $500 \mathrm{MHz}{ }^{1} \mathrm{H}$ NMR spectrum at $280 \mathrm{~K}$. The ratio clearly shows that two out of four diastereomers are predominantly formed. Although attempts to obtain crystals suitable for structure analyses failed, we speculate that diastereomers with the cobalt hydride ligand being located closely to the tert-butyl group are favored for obvious steric reasons.
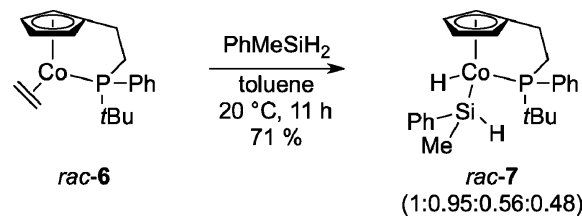

The diastereomeric mixture was fully characterized by IR and NMR spectroscopy including DEPT, HMQC, H-H-COSY and NOE experiments allowing assignments of all observed signals. The $\mathrm{Co}-\mathrm{H}$ absorptions are observed at $2043 \mathrm{~cm}^{-1}$ in contrast to $2052 \mathrm{~cm}^{-1}$ for the corresponding di-tert-butylphosphanyl complex. ${ }^{18}$ The ${ }^{31} \mathrm{P}$ NMR signals for the two dominant diastereomers appear at $\delta=93.7$ and $95.5 \mathrm{ppm}$. The ${ }^{1} \mathrm{H}$ NMR spectrum, which was obtained at $294 \mathrm{~K}$, shows the four $\mathrm{Co}-\mathrm{H}$ doublet signals at $-16.89,-16.85,-16.58$ and $-16.50 \mathrm{ppm}$ with ${ }^{2} J_{\mathrm{H}, \mathrm{P}}$ coupling constants in the range of 45.1-53.8 Hz. Variable temperature measurements in $5 \mathrm{~K}$ steps up to $350 \mathrm{~K}$ show coalescence resulting in two doublets (approx. ratio $1: 1.5$ ) with a coalescence temperature 


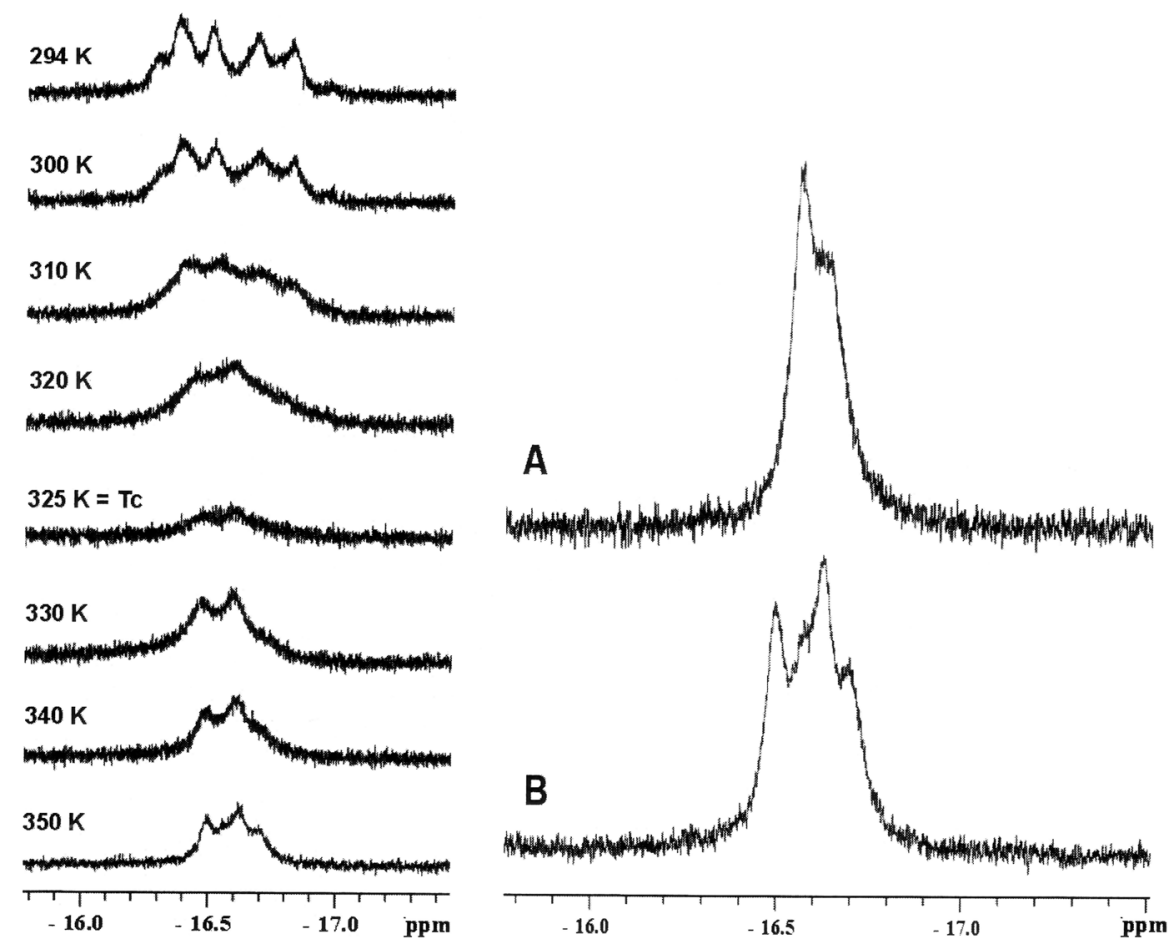

Fig. 2 Coalescence of the $\mathrm{Co}-H$ signals in the ${ }^{1} \mathrm{H}$ NMR spectrum of rac-7. Left: variable temperature measurements. Right: signal at $350 \mathrm{~K}$ with $\mathrm{P}$ decoupling (A) and with $\mathrm{P}$ coupling (B).

of $T_{\mathrm{c}}=325 \mathrm{~K}$, which corresponds to an estimated free enthalpy of activation of $64.9 \mathrm{~kJ} \mathrm{~mol}^{-1}$, which is well in the range observed for related cases. ${ }^{18}$ Fig. 2 shows the coalescence as well as the signals observed at $350 \mathrm{~K}$.

The diastereoisomerization process causing the coalescence is presumably closely related to the configurational instability of the cobalt stereogenic center in cyclopentadienylcobalt systems, which has been the subject of theoretical investigations recently published by Gandon and Aubert et al. ${ }^{46}$ According to these calculations a reductive elimination of the hydrosilane with formation of an intermediate $\eta^{2} \mathrm{Si}-\mathrm{H}$ system has to be invoked, which undergoes a change in conformation followed by oxidative re-addition. Alternatively, one might discuss a temporary decomplexation of the phosphane tether with formation of a vacant coordination site facilitating the diastereoisomerization process. The activation energies of both processes are in the same order of magnitude. ${ }^{46,47}$

The carbonylcobalt complex $\mathrm{rac}-\mathbf{8}$ corresponding to $\mathrm{rac}-\mathbf{6}$ was obtained in $65 \%$ yield by the procedure of Pályi by treatment of spiro[2.4]hepta-4,6-diene (4) with lithium tertbutylphenylphosphide followed by a THF solution of $\mathrm{Co}(\mathrm{CO})_{4} \mathrm{I}^{48}$ rac-8 complements a series of complexes with phenyl, isopropyl and tert-butyl substituents at the phosphorus atom $^{28,30}$ and is the first representative with two different substituents at this position. The carbonyl absorption of rac-8 is observed at $1894 \mathrm{~cm}^{-1}$, a value similar to that for the diphenyl $\left(1897 \mathrm{~cm}^{-1}\right)$ and the di-tert-butyl derivative $\left(1898 \mathrm{~cm}^{-1}\right){ }^{49}$

Similar to the formation of $\mathbf{r a c}-\mathbf{5}$ and $\mathrm{rac}-\mathbf{6}$ the corresponding tert-butyl(cyclohexyl) substituted complexes rac-9 and rac-10 are obtained in moderate yields from spiro[2.4]hepta-4,6-diene

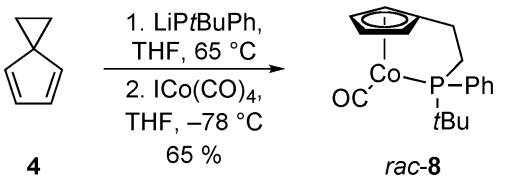

(4) by treatment with lithium tert-butylcyclohexylphosphide followed by $\mathrm{CoCl}_{2}$ and subsequent reduction with sodium amalgam in the presence of ethene. The complexes were characterized spectroscopically. According to HMQC measurements ${ }^{1} \mathrm{H}$ NMR signals at $\delta=2.34(\mathrm{~m}, 1 \mathrm{H})$ and $2.64(\mathrm{~m}, 1 \mathrm{H}) \mathrm{ppm}$ are assigned to the ethene ligand. In addition, resonances of two other ethene protons are unresolved in a broad signal at $\delta=1.87 \mathrm{ppm}$ overlapping with signals derived from the cyclohexyl substituent. The ${ }^{31} \mathrm{P}$ NMR signal at $\delta=93.2 \mathrm{ppm}$ is in accord with a completely aliphatic substitution pattern at phosphorus and resembles that of $\mathbf{2}$ $(\delta=96.4 \mathrm{ppm})$.
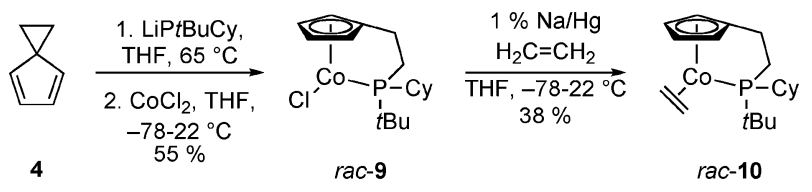

The syntheses as well as reactions of cyclopentadienyl or indenyl rhodium chelate complexes bearing a phosphane tether have been reported by Poilblanc et al., ${ }^{50}$ Tani et al., ${ }^{51-56}$ Nelson et al., ${ }^{57,58}$ Saunders et al., ${ }^{38-40,59-61}$ Hidai et al., ${ }^{62}$ Jones et al., ${ }^{63}$ Green et al., ${ }^{64}$ Salzer et al., ${ }^{65-68}$ Cole-Hamilton et al., ${ }^{69}$ Lalinde et al., ${ }^{70}$ and Whitby et al. ${ }^{71}$ In most cases the phosphane tether bears phenyl substituents, however, there are also some in which alkyl, pentafluorophenyl 
or more complicated substituents are present. To our knowledge there are no examples with tert-butyl substituents, which, according to our experience with cobalt complexes, increase the stability of the complexes and improve their crystallization properties. Therefore, and for the possibility of comparison, the di-tert-butyl substituted rhodium(I) chelate 11 was prepared by treatment of spiro[2.4]hepta-4,6-diene (4) with lithium di-tert-butylphosphide followed by $\left[\mathrm{Rh}_{2}\left(\mathrm{C}_{2} \mathrm{H}_{4}\right)_{4} \mathrm{Cl}_{2}\right]$. 11 was obtained in $72 \%$ yield as a brown oil, which is less air sensitive than the corresponding cobalt chelate 2 . The unsymmetric chelates $r a c-12$ and $r a c-13$ with tert-butylphenyl and tertbutylcyclohexyl substitution at phosphorus were prepared accordingly in $85 \%$ and $55 \%$ yield, respectively, and are also brown oils. in $d_{8}$-toluene, corresponding to energies of activation $E_{\mathrm{a}}$ of $63.0 \mathrm{~kJ} \mathrm{~mol}^{-1}$ and $62.5 \mathrm{~kJ} \mathrm{~mol}^{-1}$, respectively. ${ }^{43}$ These values are rather similar to those observed for the di-tert-butyl substituted cobalt complex $\mathbf{2}$ but significantly smaller than those observed for the tert-butylphenyl substituted cobalt analog rac-7 of rac-12.

Treatment of rac-13 with iodomethane resulted in an oxidative addition with formation of a diastereomeric mixture of rac-14 and rac-15 in 72\% yield. The diastereomeric excess (de) was $81 \%\left({ }^{1} \mathrm{H}\right.$ NMR) and could be improved to de of $95 \%$ by recrystallization from dichloromethane/hexane. Diastereoselective oxidative additions to (cyclopentadienylethyl)phosphane rhodium chelates have been investigated by the group of Kataoka and Tani, as well as by the group of Salzer. Kataoka and Tani prepared planar chiral carbonyl[1-(diphenyl-

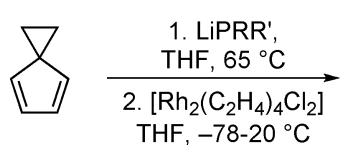

4

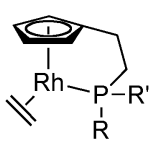

11: $\mathrm{R}=\mathrm{R}^{\prime}=t \mathrm{Bu}(72 \%)$ rac-12: $\mathrm{R}=t \mathrm{Bu}, \mathrm{R}^{\prime}=\mathrm{Ph}(85 \%)$ rac-13: $\mathrm{R}=t \mathrm{Bu}, \mathrm{R}^{\prime}=\mathrm{Cy}(55 \%)$

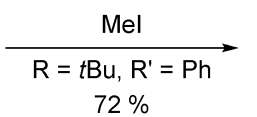

$72 \%$

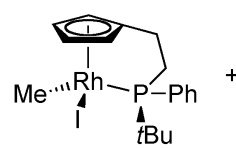

$\operatorname{rac}-14(81 \%$ de)

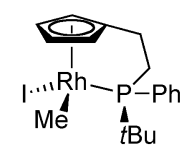

rac-15
Chelates 11-rac-13 were characterized spectroscopically. The symmetric complex 11 shows two ${ }^{1} \mathrm{H}$ NMR signals for the ethene ligand, which appear at $\delta=2.40(\mathrm{~m}, 2 \mathrm{H}) \mathrm{ppm}$ and at $\delta=2.78$ $(\mathrm{m}, 2 \mathrm{H}) \operatorname{ppm}\left[2:^{29} 1.87(\mathrm{~m}, 2 \mathrm{H}), 2.27(\mathrm{~m}, 2 \mathrm{H}) \mathrm{ppm}\right]$. The less symmetric complex rac-12 with a phenyl group at the phosphorus atom shows four signals for the ethene ligand, which appear at $\delta=2.31(\mathrm{~m}, 1 \mathrm{H}), 2.50(\mathrm{~m}, 1 \mathrm{H}), 2.93(\mathrm{~m}, 1 \mathrm{H}), 3.11$ $(\mathrm{m}, 1 \mathrm{H}) \mathrm{ppm}$. The cyclohexyl substituted chelate $\mathrm{rac}-\mathbf{1 3}$ shows a ${ }^{1} \mathrm{H}$ NMR signal at $\delta=2.86(\mathrm{~m}, 2 \mathrm{H})$ for the ethene ligand. The resonances for the other two ethene protons overlap with a large multiplet caused by the cyclohexyl protons as well as by the ethylene bridge between the cyclopentadienyl group and the phosphorus atom. The data show that the phenyl substituent has a significantly larger influence on the magnetic behavior of the ethene protons than that of the aliphatic tert-butyl or cyclohexyl substituents, an effect, which we attribute to the magnetic anisotropy of the phenyl group. The clear separation of the signals in rac-12 made it possible to determine the energy of activation of the ethene rotation by temperature dependent ${ }^{1} \mathrm{H}$ NMR spectroscopy (Fig. 3). At $400 \mathrm{MHz}$ the coalescence was observed at $T_{\mathrm{c}}=330 \mathrm{~K}$ in $d_{6}$-benzene and at $T_{\mathrm{c}}=325 \mathrm{~K}$

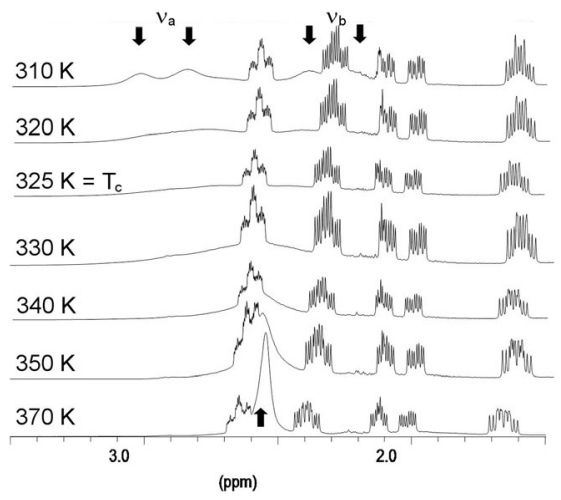

Fig. $3{ }^{1} \mathrm{H}$ NMR coalescence of $r a c-12\left(400 \mathrm{MHz}, \mathrm{C}_{6} \mathrm{D}_{6}\right)$. phosphanylethyl)indenyl]rhodium chelates and methylated these with formation of the respective acetyliodo chelates possessing metal centered chirality in addition to the planar chirality caused by the indenyl ligand. These reactions proceeded with high diastereoselectivity. ${ }^{54-56,72,73}$ The Salzer group prepared a [(diphenylphosphanyl)ethylcyclopentadienyl]ethenerhodium(I) chelate with a methyl substituent at the carbon atom next to phosphorus in enantiomerically pure form. In addition, the corresponding indenyl and fluorenyl complexes were reported. Reaction with iodomethane resulted in highly diastereoselective oxidative additions with formation of the respective iodomethyl chelate complexes. ${ }^{65,67,74}$ While Kataoka and Tani reported a stereoinduction from the planar chiral indenyl ligand to the metal center, the results of Salzer include a stereoinduction from an asymmetric carbon atom in the chelate backbone to rhodium. To our knowledge, the formation of rac-14 and rac-15 is the first case in the chemistry of cyclopentadienylalkylphosphane chelate complexes involving a stereoinduction from the asymmetric phosphorus atom to rhodium. The assignment of rac-14 as the major diastereomer is the result of NOE measurements and is in accord with an X-ray crystal structure analysis (Fig. 4).

Although the quality of the analysis is limited, the data clearly show that cyclopentadienyl carbon atoms $\mathrm{C} 1$ and $\mathrm{C} 2$ are bound significantly closer to the rhodium atom as compared to the other three cyclopentadienyl carbon atoms. This observation presumably reflects the asymmetric ligand environment around the rhodium atom and is detectable clearly, because the usual rotation around the cyclopentadienyl-rhodium axis is impossible in rac-14.

We recently reported that the nucleophilic ring opening of [4,5]benzospiro[2.4]heptadiene (16) proceeds rather slowly with $\mathrm{LiP} t \mathrm{Bu}_{2}$ (reflux in THF for $12 \mathrm{~h}$ or microwave heating at $150{ }^{\circ} \mathrm{C}$ for $40 \mathrm{~min}$ ) as compared to less bulky nucleophiles $\mathrm{LiPR}_{2}$ $(\mathrm{R}=\mathrm{Ph}, \mathrm{Cy}, \mathrm{iBu}, \mathrm{Et}) \cdot{ }^{15}$ In the context of the present work $\mathbf{1 6}$ was treated with $\mathrm{LiP} t \mathrm{BuPh}$ in THF at reflux for $3 \mathrm{~d}$ resulting in 


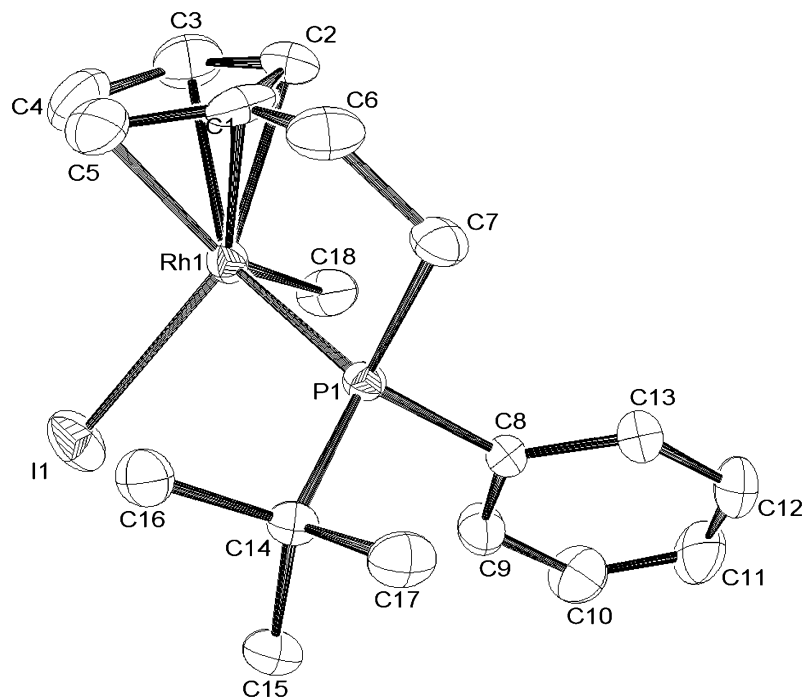

Fig. 4 Structure of $\mathrm{rac}-\mathbf{1 4}$ in the crystal. ${ }^{45}$ There are two similar molecules of rac-14 in the asymmetric unit, only one molecule is shown. Displacement ellipsoids correspond to the $50 \%$ probability level. Selected bond lengths [pm] and angles [ [ ${ }^{\circ}$ : Rh1-I1 268.59(9), Rh1-P1 227.39(14), Rh1-C1 216.7(6), Rh1-C2 214.8(5), Rh1-C3 222.8(6), Rh1-C4 226.1(7), Rh1-C5 225.0(6), Rh1-C18 217.4(5), P1-C7 185.7(5), P1-C8 181.4(5), P1-C14 188.9(5), C1-C2 142.7(9), C1-C5 143.8(10), C1-C6 149.5(9), C2-C3 139.0(10), C3-C4 133.1(11), C4-C5 138.4(11), C6-C7 150.3(9); I1-Rh1-P1 101.52(4), I1-Rh1-C18 84.26(19), P1-Rh1-C18 97.7(2), Rh1-P1-C7 100.53(19), Rh1-P1-C8 119.14(16), C7-P1-C14 105.7(2), C8-P1-C14 104.7(2).

the formation of $\mathrm{rac}-\mathbf{1 7}$. Subsequent addition of $\left[\mathrm{Rh}_{2}\left(\mathrm{C}_{2} \mathrm{H}_{4}\right)_{4} \mathrm{Cl}_{2}\right]$ gave the indenyl chelate $\mathrm{rac} \mathbf{- 1 8}$ as a diastereomeric mixture $(10: 1)$ in $71 \%$ yield. $r a c-\mathbf{1 8}$ is more air-sensitive as compared to the cyclopentadienyl chelate rac-12, so that attempts to remove a minor amount of the uncoordinated ligand were only partly successful. rac-18 was characterized spectroscopically, the NMR data reflect the asymmetry of the compound.
Nickel chelate complexes with cyclopentadienylalkylphosphane ligands have been unknown until we recently reported on the synthesis and reactivity of a number of examples with the di-tert-butyl substitution pattern at the phosphorus atom. ${ }^{15,16}$ With the new tert-butylphenyl substituted ligand in hand, we prepared the respective nickel chelate rac-20 in 70\% yield. rac-20 was characterized spectroscopically, all data are in accord with related complexes. ${ }^{15,16}$

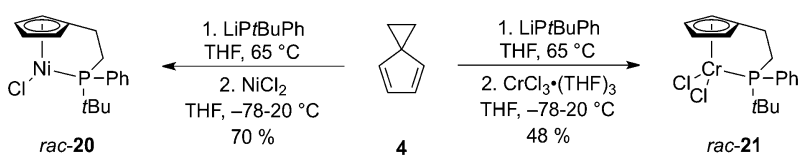

Jolly et al. reported the synthesis of cyclopentadienylchromium dichloride chelates with a phosphane tether and their application as catalysts in alkene oligo- and polymerization reactions. ${ }^{76}$ The complexes reported bear identical substituents at phosphorus, crystal structure analyses of the diphenyl and the dicyclohexyl complexes were included. We succeeded in the synthesis of the tert-butylphenyl substituted derivative, which was obtained in $48 \%$ yield by treatment of the anionic ligand with $\mathrm{CrCl}_{3} \cdot 3 \mathrm{THF}$ as blue needles from toluene. The paramagnetic complex was characterized by IR spectroscopy, mass spectrometry (including HRMS), and by an elemental analysis. These data are in accord with those obtained by Jolly for related complexes. In addition, it was possible to obtain an X-ray crystal structure analysis (Fig. 5).

The difference in $\mathrm{Cr}-\mathrm{Cl}$ bond lengths reflects the asymmetry of the complex. In this context a comparison with the closely related structures of the dicyclohexyl and the diphenyl substituted complexes is instructive. Those $\mathrm{Cr}-\mathrm{Cl}$ bond lengths are 227.53(8) and 227.86(8) pm for the dicyclohexyl but 228.37(12) and 228.44(11) pm for the diphenyl substituted complex, and the $\mathrm{Cr}-\mathrm{P}$ bond lengths also differ considerably $\left[\mathrm{PCy}_{2}:\right.$ 245.9(1), $\mathrm{PPh}_{2}:$ 244.7(1) pm]. ${ }^{76}$ In rac-21 both features are present, and consequently differences in the $\mathrm{Cr}-\mathrm{Cl}$ bond

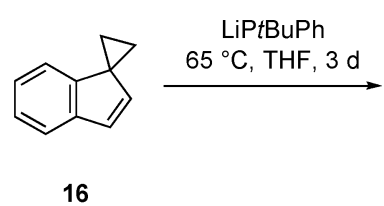

16

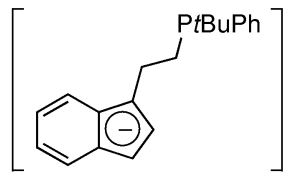

rac-17

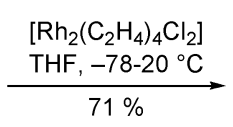

\lrcorner

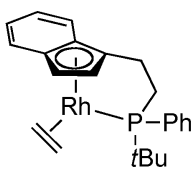

rac-18 (10:1)

As early as 1994 Poilblanc et al. reported the first synthesis of an iridium chelate with the [2-(diphenylphosphanyl)ethyl]cyclopentadienyl ligand. ${ }^{50}$ The ethene complex decomposed readily, but corresponding carbonyl or cyclooctene (coe) complexes have been isolated. ${ }^{75}$ Therefore, the iridium chelate rac-19 was prepared by reaction of the anionic ligand system with $[\mathrm{IrCl}(\mathrm{coe})]_{2}$. rac-19 was obtained in only $21 \%$ yield in addition to the residual uncoordinated ligand and was characterized spectroscopically.

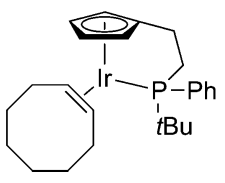

rac-19

lengths are observed. The shorter bond is next to the tert-butyl group [Cr1-Cl1, 227.4(1) pm] and the longer one next to the phenyl substituent [Cr1-Cl2, 228.7(1) pm]. The $\mathrm{Cr}-\mathrm{P}$ bond length in $r a c-21$ [246.1(1) pm] resembles more that of the dicyclohexyl [245.9(1) pm] than that of the diphenyl compound [244.7(1) pm]. It seems reasonable that these data reflect the steric bulk of the tert-butyl substituent as compared to that of the phenyl group.

In conclusion, we have reported the first chelate complexes with the [2-(tert-butylphenylphosphanyl)ethyl]cyclopentadienyl ligand system, in which the phosphorus atom is a center of chirality. This asymmetry is reflected in the structures of the complexes, three of which have been determined, as well as in the diastereoselectivity of the reaction of rhodium chelate rac-12 with iodomethane. While stereoinduction from an 


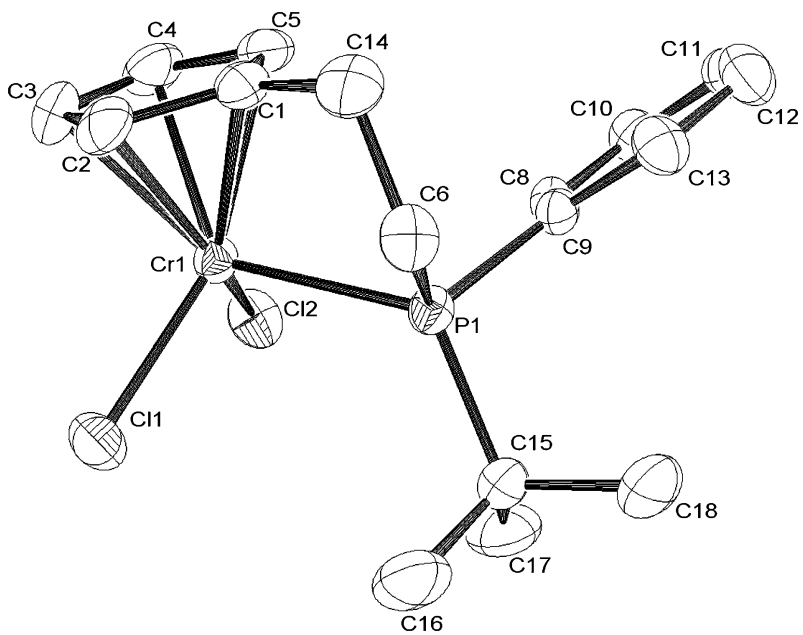

Fig. 5 Structure of rac-21 in the crystal. ${ }^{45}$ There are two similar molecules of rac-21 in the asymmetric unit, only one molecule is shown. Displacement ellipsoids correspond to the $50 \%$ probability level. Selected bond lengths [pm] and angles [ ${ }^{\circ}$ : $\mathrm{Cr} 1-\mathrm{Cl} 1$ 227.39(10), Cr1-Cl2 228.71(11), Cr1-P1 246.13(11), Cr1-C1 224.5(3), Cr1-C2 222.3(4), Cr1-C3 222.6(4), Cr1-C4 222.3(3), Cr1-C5 222.4(3), P1-C6 182.9(3), P1-C9 181.5(3), P1-C15 186.1(3), C1-C2 138.8(4), C1-C5 141.9(4), C1-C14 150.2(4), C2-C3 138.7(5), C3-C4 137.4(5), C4-C5 141.0(4), C6-C14 152.6(5); Cl1-Cr1-Cl2 98.27(4), Cl1-Cr1-P1 98.91(4), Cl2-Cr 101.18(4).

indenyl ligand or an asymmetric carbon atom in the chelate backbone has earlier been reported, this is the first case that the asymmetric phosphorus atom causes this diastereoselectivity.

\section{Experimental section}

General: All manipulations involving air sensitive material were performed in flame-dried reaction vessels in an argon or nitrogen atmosphere using vacuum line and standard Schlenk techniques. Spiro[2.4]hepta-4,6-diene (4) and [4,5]benzospiro[2.4]hepta-4,6-diene (16) were prepared according to published procedures. ${ }^{53,77}$ Diethyl ether (EE), and THF were distilled from sodium benzophenone ketyl. Hexane, pentane and dichloromethane were dried with calcium hydride and freshly distilled before use. Petroleum ether (PE) was dried with calcium chloride. All the solvents were purged with nitrogen before use. Column chromatography was carried out by flash chromatography. ${ }^{78}$ Silica gel (J. T. Baker, $\left.40 \mu \mathrm{m}\right)$ was degassed three times by heating it with a flame at reduced pressure followed by setting it at normal pressure with nitrogen. IR-Spectra: Bruker FT-IR spectrometer Vektor 22 (ATR). Mass spectra: Finnegan MAT 112 and MAT 312. HRMS $(E S I)$ : Micromass LCT with a lock spray ion source combined with a Water Alliances 2695 HPLC unit; VG autospec (peakmatching method, PFK). ${ }^{1} H$ NMR: Bruker AVS 200 (200.1 $\mathrm{MHz})$, AVS $400(400.1 \mathrm{MHz})$ and DRX $500(500.1 \mathrm{MHz})$. ${ }^{13} C$ NMR: Bruker AVS $200(50.3 \mathrm{MHz})$ and AVS 400 $(100.6 \mathrm{MHz})$. Signal multiplicities were determined with ATP and DEPT techniques. ${ }^{31} P$ NMR: Bruker AVS 400 (161.9 MHz). Melting points: Electrothermal IA9000 Series Digital Melting Point Apparatus. Elemental analyses: Elementar Vario EL.
Chloro- $\left\{\eta^{5}: \eta^{1}[2-(\right.$ tert-butylphenylphosphanyl)ethyl]cyclopentadienyl\}cobalt(II) (rac-5)

At $-78{ }^{\circ} \mathrm{C}$ butyllithium in hexane $(19.4 \mathrm{~mL}, 31.2 \mathrm{mmol}$, $1.6 \mathrm{M})$ was added dropwise to tert-butylphenylphosphane (4.314 g, $25.9 \mathrm{mmol})$ in THF (100 mL). After stirring for $2 \mathrm{~h}$ at $20{ }^{\circ} \mathrm{C}$ spiro[2.4]hepta-4,6-diene (4, $\left.2.484 \mathrm{~g}, 27.0 \mathrm{mmol}\right)$ was added, and after stirring for $4 \mathrm{~h}$ at $65{ }^{\circ} \mathrm{C}$ the mixture was cooled to $-78{ }^{\circ} \mathrm{C}$, and $\mathrm{CoCl}_{2}(4.050 \mathrm{~g}, 31.2 \mathrm{mmol})$ was added. The mixture was slowly warmed to $20{ }^{\circ} \mathrm{C}$ and stirred for another $1 \mathrm{~h}$. After solvent removal at reduced pressure the residue was taken up with diethyl ether $(25 \mathrm{~mL})$ and filtered through a $3 \mathrm{~cm}$ thick layer of Celite. After solvent removal the product was isolated by column chromatography $(3 \times 20 \mathrm{~cm}$, TBME) to give $5(5.960 \mathrm{~g}, 16.9 \mathrm{mmol}, 65 \%)$ as a purple solid, mp $106.1{ }^{\circ} \mathrm{C}$.

IR: $\tilde{v}=3406(\mathrm{w}), 3078(\mathrm{~m}), 2940$ (s, C-H), $2863(\mathrm{w}, \mathrm{C}-\mathrm{H})$, 2360 (w), 1630 (m), 1473 (w), 1461 (m), 1437 (w, P-Ph), 1365 $(\mathrm{w}, t-\mathrm{Bu}), 1183(\mathrm{~m}), 1163(\mathrm{w}), 1101(\mathrm{w}), 1035(\mathrm{~m}), 1023(\mathrm{~s}$, $t$-Bu), $934(\mathrm{~m}), 848$ (w), 806 (s, Cp), 755 (m), 700 (s), $621(\mathrm{~m}) \mathrm{cm}^{-1}$. MS: $m / z(\%)=351(20)\left[\mathrm{M}^{+}\right], 259(54)\left[\mathrm{M}^{+}-\mathrm{CoCl}\right]$, 182 (17), 160 (40), 126 (65), 110 (62), 79 (76), 57 (19) $\left[t-\mathrm{Bu}^{+}\right]$. HRMS $\left(\mathrm{M}^{+}=\mathrm{C}_{17} \mathrm{H}_{22} \mathrm{ClCoP}\right)$ calcd 351.0482, found 351.0479. Anal. $\left(\mathrm{C}_{17} \mathrm{H}_{22} \mathrm{ClCoP}\right)$ calcd $\mathrm{C} 50.01 ; \mathrm{H}$ 7.27, found C 50.14 ; H 7.19 .

$\left\{\eta^{5}: \eta^{1}[2-(\right.$ tert-Butylphenylphosphanyl)ethyl]cyclopentadienyl $\}$ ( $\eta^{2}$-ethen)-cobalt(I) (rac-6)

At $-78{ }^{\circ} \mathrm{C}$ sodium amalgam $(1 \%, 3.2 \mathrm{~mL}, 2.1 \mathrm{mmol})$ was added to chlorocobalt complex rac-5 $(0.765 \mathrm{~g}, 4.3 \mathrm{mmol})$ in THF $(15 \mathrm{~mL})$, and ethene was purged through the mixture. After $1 \mathrm{~h}$ the mixture was slowly warmed to $20{ }^{\circ} \mathrm{C}$ and stirred for another $4 \mathrm{~h}$. After separation of the organic layer from the amalgam the solvent was removed at reduced pressure, and the residue was taken up with hexane $(15 \mathrm{~mL})$ and filtered through a frit covered with a $3 \mathrm{~cm}$ thick layer of Celite. After concentration at reduced pressure the filtrate was cooled to $-25{ }^{\circ} \mathrm{C}$ affording complex rac-6 (1.19 g, $\left.3.5 \mathrm{mmol}, 40 \%\right)$ as brown-black crystals $\left(\mathrm{mp} 73.0{ }^{\circ} \mathrm{C}\right)$, purity $\left({ }^{1} \mathrm{H}\right.$ NMR $) \geq 95 \%$.

IR: $\tilde{v}=3052\left(\mathrm{~m}_{2} \mathrm{C}_{2} \mathrm{H}_{4}\right), 2959(\mathrm{~s}, \mathrm{C}-\mathrm{H}), 2862(\mathrm{~m}, \mathrm{C}-\mathrm{H}), 2364$ (w), 1474 (m, C-H), 1461 (m), 1434 (m, P-Ph), 1362 (m, $t$-Bu), $1261\left(\mathrm{~m}, \mathrm{C}_{2} \mathrm{H}_{4}\right), 1164(\mathrm{~m}), 1095(\mathrm{~m}), 1027$ (m, $t$-Bu), 895 (w), 800 (s, Cp-R), 745 (m), 699 (s) $\mathrm{cm}^{-1}$. ${ }^{1} \mathrm{H}-\mathrm{NMR}(400 \mathrm{MHz}$, $\left.\mathrm{C}_{6} \mathrm{D}_{6}\right): \delta=0.76\left(\mathrm{~d},{ }^{3} J_{\mathrm{H}, \mathrm{P}}=12.7 \mathrm{~Hz}, 9 \mathrm{H}, \mathrm{CH}_{3}\right), 1.46(\mathrm{~m}, 1 \mathrm{H}$, $\left.\mathrm{PCH}_{2} \mathrm{CH}_{2}\right), 1.79-1.96\left(\mathrm{~m}, 1 \mathrm{H}, \mathrm{PCH}_{2} \mathrm{CH}_{2}\right), 2.03(\mathrm{~m}, 2 \mathrm{H}$, $\left.\mathrm{PCH}_{2}\right), 2.05\left(\mathrm{~m}, 2 \mathrm{H}, \mathrm{CH}_{2}=\mathrm{CH}_{2}\right), 2.44\left(\mathrm{~m}, 1 \mathrm{H}, \mathrm{CH}_{2}=\mathrm{CH}_{2}\right)$, $2.73\left(\mathrm{~m}, 1 \mathrm{H}, \mathrm{CH}_{2}=\mathrm{CH}_{2}\right), 3.54\left(\mathrm{~s}, 1 \mathrm{H}, \mathrm{PCH}_{2} \mathrm{CH}_{2} \mathrm{CCHCH}\right)$, $3.81\left(\mathrm{~s}, 1 \mathrm{H}, \mathrm{PCH}_{2} \mathrm{CH}_{2} \mathrm{CCH}\right), 5.05\left(\mathrm{~s}, 1 \mathrm{H}, \mathrm{PCH}_{2} \mathrm{CH}_{2} \mathrm{CCH}\right)$, $5.55\left(\mathrm{~s}, 1 \mathrm{H}, \mathrm{PCH}_{2} \mathrm{CH}_{2} \mathrm{CCHCHCH}\right), 7.15\left(\mathrm{~d},{ }^{2} J_{\mathrm{H}, \mathrm{P}}=6.0 \mathrm{~Hz}\right.$, $3 \mathrm{H}, o-, p-\mathrm{CH}), 7.98\left(\mathrm{t},{ }^{3} J_{\mathrm{H}, \mathrm{P}}=7.5 \mathrm{~Hz}, 2 \mathrm{H}, m-\mathrm{CH}\right) \mathrm{ppm}$. ${ }^{13} \mathrm{C}-\mathrm{NMR}\left(100.6 \mathrm{MHz}, \mathrm{C}_{6} \mathrm{D}_{6}\right): \delta=22.1\left(\mathrm{~d},{ }^{2} J_{\mathrm{C}, \mathrm{P}}=5.5 \mathrm{~Hz}\right.$, $\left.\mathrm{PCH}_{2} \mathrm{CH}_{2}\right), 22.9\left(=\mathrm{CH}_{2}\right), 26.5\left(\mathrm{~d},{ }^{2} J_{\mathrm{C}, \mathrm{P}}=3.7 \mathrm{~Hz}, \mathrm{CH}_{3}\right), 31.2$ $\left(\mathrm{d},{ }^{1} J_{\mathrm{C}, \mathrm{P}}=14.1 \mathrm{~Hz}, \mathrm{PCCH} 3\right), 38.8\left(\mathrm{~d},{ }^{1} J_{\mathrm{C}, \mathrm{P}}=24.9 \mathrm{~Hz}, \mathrm{PCH}_{2}\right)$, $77.7\left(\mathrm{PCH}_{2} \mathrm{CH}_{2} \mathrm{CCHCH}\right), \quad 79.1\left(\mathrm{~d},{ }^{4} J_{\mathrm{C}, \mathrm{P}}=5.1 \mathrm{~Hz}\right.$, $\left.\mathrm{PCH}_{2} \mathrm{CH}_{2} \mathrm{CCH}\right), 82.4\left(\mathrm{PCH}_{2} \mathrm{CH}_{2} \mathrm{CCHCH}\right), 83.0\left(\mathrm{~d},{ }^{4} J_{\mathrm{C}, \mathrm{P}}=\right.$ $\left.5.3 \mathrm{~Hz}, \quad \mathrm{PCH}_{2} \mathrm{CH}_{2} \mathrm{CCH}\right), 108.8\left(\mathrm{~d},{ }^{3} J_{\mathrm{C}, \mathrm{P}}=6.3 \mathrm{~Hz}\right.$, $\left.\mathrm{PCH}_{2} \mathrm{CH}_{2} C\right), 127.6\left(\mathrm{~d},{ }^{2} J_{\mathrm{C}, \mathrm{P}}=8.3 \mathrm{~Hz}, o-\mathrm{CH}\right), 129(p-\mathrm{CH})$, $134.0\left(\mathrm{~d},{ }^{3} J_{\mathrm{C}, \mathrm{P}}=9.1 \mathrm{~Hz}, m-\mathrm{CH}\right), 135.4\left(\mathrm{~d},{ }^{1} J_{\mathrm{C}, \mathrm{P}}=20.3 \mathrm{~Hz}\right.$, $\mathrm{PCCH})$ ppm. ${ }^{31} \mathrm{P}-\mathrm{NMR}\left(162 \mathrm{MHz}, \mathrm{C}_{6} \mathrm{D}_{6}\right): \delta=79.9 \mathrm{ppm}$. 
MS: $m / z(\%)=344\left[\mathrm{M}^{+}\right](11), 316\left[\mathrm{M}^{+}-\mathrm{CH}_{2}=\mathrm{CH}_{2}\right](100)$, 259 (86), 180 (43), 136 (61), 91 (24), 57 (41). HRMS ( ${ }^{+}=$ $\left.\mathrm{C}_{19} \mathrm{H}_{26} \mathrm{CoP}\right)$ calcd 344.1104, found 344.1102. $T_{\mathrm{c}}=350 \mathrm{~K}$ ( $d_{8}$-toluene, $400 \mathrm{MHz}$ ), $\Delta G^{\ddagger} \approx 68.7 \mathrm{~kJ} \mathrm{~mol}^{-1}$.

Crystal structure analysis of $\mathrm{rac}-6 \mathbf{6}^{45}$ empirical formula $\mathrm{C}_{19} \mathrm{H}_{26} \mathrm{CoP}$, molecular weight 344.30 , crystal system monoclinic, space group $P 2_{1} / n, a=11.070(5), b=11.504(4)$, $c=14.202(6) \AA, \alpha=90.00^{\circ}, \beta=104.47(5)^{\circ}, \gamma=90.00^{\circ}$, $V=1751.3(12) \AA^{3}, Z=4, d_{\text {calcd }}=1.306 \mathrm{~g} \mathrm{~cm}^{-1}, F(000)=$ $728, \mu=1.063 \mathrm{~mm}^{-1}$, Stoe IPDS diffractometer, $T=294 \mathrm{~K}$, $\operatorname{MoK}_{\alpha}(\lambda=0.71073 \AA), \theta_{\text {min }}=2.10^{\circ}, \theta_{\max }=26.23^{\circ}, 24177$ measured reflections $(-13 \leq h \leq 13,-14 \leq k \leq 14,-17 \leq$ $l \leq 17), 3479$ independent, 2070 observed reflections, $R_{\text {int }}=$ $0.089, R=0.0301, \mathrm{w} R=0.0534$, residual electron density 0.244 and $-0.286 \mathrm{e}^{-3}$, Gof $=0.803$, refinement program SHELXL-97, $N_{\text {ref }}=3479, N_{\text {par }}=202$.

$\left\{\eta^{5}: \eta^{1}[2-(\right.$ tert-Butylphenylphosphanyl)ethyl]cyclopentadienyl $\}-$ (hydrido)(methylphenylsilyl)cobalt(III) (rac-7)

Methylphenylsilane $(0.5 \mathrm{~mL}, 4.0 \mathrm{mmol})$ was added to ethenecobalt chelate rac-6 $(465 \mathrm{mg}, 1.4 \mathrm{mmol})$ in toluene $(14 \mathrm{~mL})$. After stirring for $11 \mathrm{~h}$ at $60{ }^{\circ} \mathrm{C}$ the solvent was removed at reduced pressure, and the residue was taken up with hexane $(5 \mathrm{~mL})$ and filtered through a frit covered with a $3 \mathrm{~cm}$ thick layer of Celite. The solvent was removed at reduced pressure to give rac-7 (420 mg, $1.35 \mathrm{mmol}, 71 \%)$ as a yellow oil as a mixture of four diastereomers $[1.00: 0.95: 0.56: 0.48$, purity $\geq 95 \%\left({ }^{1} \mathrm{H}\right.$ NMR $\left.)\right]$.

IR: $\tilde{v}=3060(\mathrm{w}), 2956$ (m, C-H), 2043 (m, Co-H), $1946(\mathrm{w}$, Si-H), 1461 (w, C-H), 1426 (m, P-Ph), 1392 (w), 1363 (w, $t$-Bu), $1310(\mathrm{w}), 1260(\mathrm{~m}), 1232$ (w), 1181 (w), 1123 (w), $1095(\mathrm{~m}, \mathrm{C}-\mathrm{H})$, 1014 (s), 996 (m), 878 (m), 816 (s, Si-H), $730(\mathrm{~m}), 696(\mathrm{~s}) \mathrm{cm}^{-1}$. ${ }^{1} \mathrm{H}-\mathrm{NMR}\left(400 \mathrm{MHz}, \mathrm{C}_{6} \mathrm{D}_{6}\right): \delta=-16.89\left(\mathrm{~d},{ }^{2} J_{\mathrm{H}, \mathrm{P}}=53.8 \mathrm{~Hz}\right.$, $1 / 4 \mathrm{H}, 14-\mathrm{H}),-16.85\left(\mathrm{~d},{ }^{2} J_{\mathrm{H}, \mathrm{P}}=45.1 \mathrm{~Hz}, 1 / 4 \mathrm{H}, 14-\mathrm{H}\right),-16.58$ $\left(\mathrm{d},{ }^{2} J_{\mathrm{H}, \mathrm{P}}=53.1 \mathrm{~Hz}, 1 / 4 \mathrm{H}, 14-\mathrm{H}\right),-16.50\left(\mathrm{~d},{ }^{2} J_{\mathrm{H}, \mathrm{P}}=46.1 \mathrm{~Hz}\right.$, $1 / 4 \mathrm{H}, 14-\mathrm{H}), 0.37\left(\mathrm{~s}, 3 \mathrm{H}, \mathrm{SiCH}_{3}\right), 0.87\left(\mathrm{~m}, 9 \mathrm{H}, \mathrm{CCH}_{3}\right)$, 1.66-2.07 (m, 2H, $\left.\mathrm{PCH}_{2} \mathrm{CH}_{2}\right), 1.42-2.58\left(\mathrm{~m}, 2 \mathrm{H}, \mathrm{PCH}_{2}\right)$, 4.26-5.15 (m, 4H, Cp-H), 5.59 (s, 1H, Si-H), 7.07-7.58 (m, 10H, Ph-H) ppm. ${ }^{13} \mathrm{C}-\mathrm{NMR}\left(100.6 \mathrm{MHz}, \mathrm{C}_{6} \mathrm{D}_{6}\right.$, main product $): \delta=1.4\left(\mathrm{SiCH}_{3}\right), 22.5\left(\mathrm{~d},{ }^{2} J_{\mathrm{C}, \mathrm{P}}=5.2 \mathrm{~Hz}\right.$, $\left.\mathrm{PCH}_{2} \mathrm{CH}_{2}\right), 26.5\left(\mathrm{~d},{ }^{2} J_{\mathrm{C}, \mathrm{P}}=3.6 \mathrm{~Hz}, \mathrm{CCH}_{3}\right), 31.9(\mathrm{~d}$, $\left.{ }^{1} J_{\mathrm{C}, \mathrm{P}}=9.5 \mathrm{~Hz}, C \mathrm{CH}_{3}\right), 40.5\left(\mathrm{~d},{ }^{1} J_{\mathrm{C}, \mathrm{P}}=24.7 \mathrm{~Hz}, \mathrm{PCH}_{2}\right)$, $80.2\left(\mathrm{C}_{\mathrm{Cp}} \mathrm{CH}\right), 80.8\left(\mathrm{C}_{\mathrm{Cp}} \mathrm{CH}\right), 80.9\left(\mathrm{C}_{\mathrm{Cp}} \mathrm{CH}\right), 84.2\left(\mathrm{C}_{\mathrm{Cp}} \mathrm{CH}\right)$, $116.0\left(\mathrm{~d},{ }^{3} J_{\mathrm{C}, \mathrm{P}}=6.7 \mathrm{~Hz}, C_{\mathrm{Cp}} \mathrm{CH}_{2}\right), 127.6\left(\mathrm{~d},{ }^{2} J_{\mathrm{C}, \mathrm{P}}=5.3 \mathrm{~Hz}\right.$, P-o- $\left.\mathrm{C}_{\mathrm{Ph}} \mathrm{H}\right), \quad 127.9 \quad\left(\mathrm{Si}_{-} \mathrm{C}_{\mathrm{Ph}} \mathrm{H}\right), \quad 129.4 \quad\left(\mathrm{P}-p-\mathrm{C}_{\mathrm{Ph}} \mathrm{H}\right), \quad 132.7$ $\left(\mathrm{Si}_{-} \mathrm{C}_{\mathrm{Ph}} \mathrm{H}\right), 133.5\left(\mathrm{~d},{ }^{3} J_{\mathrm{C}, \mathrm{P}}=9.1 \mathrm{~Hz}, \mathrm{P}-m-\mathrm{C}_{\mathrm{Ph}} \mathrm{H}\right), 134.7$ $\left(\mathrm{Si}-\mathrm{C}_{\mathrm{Ph}} \mathrm{H}\right), 135.0\left(\mathrm{PC}_{\mathrm{Ph}} \mathrm{C}\right), 148.6\left(\mathrm{Si}_{\mathrm{Ph}} \mathrm{C}\right)$ ppm. ${ }^{31} \mathrm{P}-\mathrm{NMR}$ $\left(162 \mathrm{MHz}, \mathrm{C}_{6} \mathrm{D}_{6}\right): \delta=93.7,95.5$ ppm. MS: $m / z(\%)=437$ (32) $\left[\mathrm{M}^{+}-\mathrm{H}\right], 436(100)\left[\mathrm{M}^{+}-2 \mathrm{H}\right], 358(31), 258(42), 121$ (58), 105 (39), 78 (70), 57 (78) $\left[t-\mathrm{Bu}^{+}\right]$. HRMS $\left(\mathrm{M}^{+}-2 \mathrm{H}=\right.$ $\mathrm{C}_{24} \mathrm{H}_{30} \mathrm{CoPSi}$ ) calcd 436.1186 , found 436.1182 .

\section{Carbonyl $\left\{\eta^{5}: \eta^{1}[2-(\right.$ tert-butylphenylphosphanyl)ethyl]- cyclopentadienyl\}cobalt(I) ( rac-8)}

A solution of $\mathrm{ICo}(\mathrm{CO})_{4}$ was prepared by addition of $\mathrm{Co}_{2}(\mathrm{CO})_{8}$ $(1.000 \mathrm{~g}, 2.9 \mathrm{mmol})$ in THF $(15 \mathrm{~mL})$ to $\mathrm{I}_{2}(0.740 \mathrm{~g}, 2.9 \mathrm{mmol})$ in THF $(25 \mathrm{~mL})$ at $-78{ }^{\circ} \mathrm{C}$ and subsequent stirring for $1 \mathrm{~h}$ at $-78{ }^{\circ} \mathrm{C} .{ }^{48} \mathrm{~A}$ solution of the anionic ligand was prepared by addition at $-78{ }^{\circ} \mathrm{C}$ of butyllithium in hexane $(3.2 \mathrm{~mL}, 5.1 \mathrm{mmol}$, $1.6 \mathrm{M})$ to tert-butylphenylphosphane $(0.719 \mathrm{~g}, 4.3 \mathrm{mmol})$ in THF $\left(15 \mathrm{~mL}\right.$ ), followed by stirring for $2 \mathrm{~h}$ at $20{ }^{\circ} \mathrm{C}$ and addition of spiro[2.4]hepta-4,6-diene $(4,0.45 \mathrm{~mL}, 4.5 \mathrm{mmol})$. This solution was added to the $\mathrm{ICo}(\mathrm{CO})_{4}$ solution at $-78{ }^{\circ} \mathrm{C}$, and the mixture was stirred for $1 \mathrm{~h}$ at $-78{ }^{\circ} \mathrm{C}$ and then for $4 \mathrm{~h}$ at $20^{\circ} \mathrm{C}$. After solvent removal at reduced pressure the residue was taken up with hexane $(15 \mathrm{~mL})$ and filtered through a frit covered with a $3 \mathrm{~cm}$ thick layer of Celite. Solvent removal from the filtrate afforded complex rac-8 $(0.667 \mathrm{~g}, 1.9 \mathrm{mmol}, 45 \%)$ as a brown-black oil (purity $\geq 90 \%,{ }^{1} \mathrm{H}$ NMR).

IR: $\tilde{v}=2961$ (m, C-H), 2077 (w), 2015 (s, impur.), 1946 (s, impur.), 1894 (CO, s), 1620 (w), 1474 (w, C-H), 1435 (w, P-Ph), 1166 (w), 1099 (w), 1015 (w, $t$-Bu), 815 (w, Cp), 746 (w), 697 (m) $\mathrm{cm}^{-1} .{ }^{1} \mathrm{H}-\mathrm{NMR}\left(400 \mathrm{MHz}, \mathrm{C}_{6} \mathrm{D}_{6}\right): \delta=0.9\left(\mathrm{~d},{ }^{3} J_{\mathrm{H}, \mathrm{P}}=14.3 \mathrm{~Hz}\right.$, $\left.\mathrm{CH}_{3}\right), 1.37\left(\mathrm{~m}, 1 \mathrm{H}, \mathrm{PCH}_{2} \mathrm{CH}_{2}\right), 1.72\left(\mathrm{~m}, 1 \mathrm{H}, \mathrm{PCH}_{2} \mathrm{CH}_{2}\right), 2.30$ (m, $\left.2 \mathrm{H}, \mathrm{PCH}_{2}\right), 4.62\left(\mathrm{~s}, 1 \mathrm{H}, \mathrm{PCH}_{2} \mathrm{CH}_{2} \mathrm{CCHCH}\right), 4.89(\mathrm{~s}, 1 \mathrm{H}$, $\left.\mathrm{PCH}_{2} \mathrm{CH}_{2} \mathrm{CCHCH}\right), 4.97\left(\mathrm{~s}, 1 \mathrm{H}, \mathrm{PCH}_{2} \mathrm{CH}_{2} \mathrm{CCH}\right), 5.81(\mathrm{~s}, 1 \mathrm{H}$, $\left.\mathrm{PCH}_{2} \mathrm{CH}_{2} \mathrm{CCH}\right), 7.08(\mathrm{~m}, 3 \mathrm{H}, o-\mathrm{CH}, p-\mathrm{CH}), 7.79\left(\mathrm{t},{ }^{3} J_{\mathrm{H}, \mathrm{P}}=\right.$ $9.5 \mathrm{~Hz}, 2 \mathrm{H}, m-\mathrm{CH}) \mathrm{ppm} .{ }^{13} \mathrm{C}-\mathrm{NMR}\left(100.6 \mathrm{MHz}, \mathrm{C}_{6} \mathrm{D}_{6}\right): \delta=23.2$ $\left(\mathrm{d},{ }^{2} J_{\mathrm{C}, \mathrm{P}}=6.5 \mathrm{~Hz}, \mathrm{PCH}_{2} \mathrm{CH}_{2}\right), 26.7\left(\mathrm{~d},{ }^{2} J_{\mathrm{C}, \mathrm{P}}=4.7 \mathrm{~Hz}, \mathrm{CH}_{3}\right)$, $32.1\left(\mathrm{~d},{ }^{1} J_{\mathrm{C}, \mathrm{P}}=25.1 \mathrm{~Hz}, \mathrm{CCH}_{3}\right), 39.5\left(\mathrm{~d},{ }^{1} J_{\mathrm{C}, \mathrm{P}}=24.7 \mathrm{~Hz}, \mathrm{PCH}_{2}\right)$, $78.6\left(\mathrm{~d},{ }^{4} J_{\mathrm{C}, \mathrm{P}}=3.6 \mathrm{~Hz}, \mathrm{PCH}_{2} \mathrm{CH}_{2} \mathrm{CCH}\right), 80.5\left(\mathrm{~d},{ }^{5} J_{\mathrm{C}, \mathrm{P}}=1.1 \mathrm{~Hz}\right.$, $\left.\mathrm{PCH}_{2} \mathrm{CH}_{2} \mathrm{CCHCH}\right), 81.1\left(\mathrm{~d},{ }^{4} J_{\mathrm{C}, \mathrm{P}}=3.6 \mathrm{~Hz}, \mathrm{PCH}_{2} \mathrm{CH}_{2} \mathrm{CCH}\right)$, $83.8\left(\mathrm{~d},{ }^{5} J_{\mathrm{C}, \mathrm{P}}=1.1 \mathrm{~Hz}, \mathrm{PCH}_{2} \mathrm{CH}_{2} \mathrm{CCHCH}\right), 108.7\left(\mathrm{~d},{ }^{3} J_{\mathrm{C}, \mathrm{P}}=\right.$ $\left.6.5 \mathrm{~Hz}, \mathrm{PCH}_{2} \mathrm{CH}_{2} \mathrm{C}\right), 127.8\left(\mathrm{~d},{ }^{2} J_{\mathrm{C}, \mathrm{P}}=5.1 \mathrm{~Hz}, o-\mathrm{CH}\right), 129.7(\mathrm{~d}$, $\left.{ }^{4} J_{\mathrm{C}, \mathrm{P}}=2.3 \mathrm{~Hz}, p-\mathrm{CH}\right), 133.7\left(\mathrm{~d},{ }^{3} J_{\mathrm{C}, \mathrm{P}}=10.1 \mathrm{~Hz}, m-\mathrm{CH}\right), 134.2$ $\left(\mathrm{d},{ }^{1} J_{\mathrm{C}, \mathrm{P}}=31.2 \mathrm{~Hz}, \mathrm{PCCH}\right), 207.1(\mathrm{CO}) \mathrm{ppm} .{ }^{31} \mathrm{P}-\mathrm{NMR}$ (162 $\left.\mathrm{MHz}, \mathrm{C}_{6} \mathrm{D}_{6}\right): \delta=108.7 \mathrm{ppm}$. MS: $m / z(\%)=344(34)\left[\mathrm{M}^{+}\right], 316$ (96) $\left[\mathrm{M}^{+}-\mathrm{CO}\right], 260$ (100), 259 (65), 180 (28), 136 (44), 58 (24). HRMS $\left(\mathrm{M}^{+}=\mathrm{C}_{18} \mathrm{H}_{22} \mathrm{CoOP}\right)$ calcd 344.0740, found 344.0741.

\section{Chloro- $\left\{\eta^{5}: \eta^{1}[2-(\right.$ tert-butylcyclohexylphosphanyl)ethyl]- cyclopentadienyl\}cobalt(II) (rac-9)}

At $-78{ }^{\circ} \mathrm{C}$ butyllithium in hexane $(6.4 \mathrm{~mL}, 10.2 \mathrm{mmol}, 1.6 \mathrm{M})$ was added dropwise to tert-butylphenylphosphane $(1.489 \mathrm{~g}$, $8.7 \mathrm{mmol})$ in THF $(30 \mathrm{~mL})$. After stirring for $2 \mathrm{~h}$ at $20^{\circ} \mathrm{C}$ spiro[2.4]hepta-4,6-diene $(\mathbf{4}, 0.9 \mathrm{~mL}, 9.0 \mathrm{mmol})$ was added, and after stirring for $2 \mathrm{~h}$ at $65{ }^{\circ} \mathrm{C}$ the mixture was cooled to $-78{ }^{\circ} \mathrm{C}$, and $\mathrm{CoCl}_{2}(1.350 \mathrm{~g}, 10.4 \mathrm{mmol})$ was added. The mixture was slowly warmed to $20{ }^{\circ} \mathrm{C}$ and stirred for another $2 \mathrm{~h}$. After solvent removal at reduced pressure the residue was taken up with diethyl ether $(15 \mathrm{~mL})$ and filtered through a frit covered with a $3 \mathrm{~cm}$ thick layer of Celite. The filtrate was concentrated and cooled at $-25{ }^{\circ} \mathrm{C}$ affording complex rac-9 $(1.717 \mathrm{~g}, 4.8 \mathrm{mmol}, 55 \%)$ as a purple solid $\left(\mathrm{mp} 113{ }^{\circ} \mathrm{C}\right)$.

IR: $\tilde{v}=2922(\mathrm{~s}, \mathrm{C}-\mathrm{H}), 2851(\mathrm{~m}, \mathrm{C}-\mathrm{H}), 2357$ (w), $1693(\mathrm{w})$, $1446(\mathrm{~m}), 1394(\mathrm{w}), 1365(\mathrm{~m}, t-\mathrm{Bu}), 1262(\mathrm{w}), 1103(\mathrm{~m}), 1031(\mathrm{~m})$, $1016(\mathrm{~m}, t-\mathrm{Bu}), 936(\mathrm{w}), 888(\mathrm{w}), 851(\mathrm{w}), 828(\mathrm{~m}), 796(\mathrm{~s}, \mathrm{Cp})$, $735(\mathrm{w}) \mathrm{cm}^{-1}$. MS: $m / z(\%)=357(100)\left[\mathrm{M}^{+}\right], 263(82)$ [M $\left.\mathrm{M}^{+}-\mathrm{CoCl}\right], 258$ (49), 182 (90), 163 (44), 57 (34). HRMS $\left(\mathrm{M}^{+}=\mathrm{C}_{17} \mathrm{H}_{28} \mathrm{ClCoP}\right)$ calcd 357.0949, found 357.0949.

\section{$\left\{\eta^{5}: \eta^{1}[2-(\right.$ tert-Butylcyclohexylphosphanyl)ethyl]- cyclopentadienyl $\}$ - $\left(\boldsymbol{\eta}^{2}\right.$-ethen)-cobalt(I) (rac-10)}

At $-78{ }^{\circ} \mathrm{C}$ sodium amalgam $(1 \%, 6.3 \mathrm{~mL}, 3.7 \mathrm{mmol})$ was added to chlorocobalt complex rac-9 $(1.171 \mathrm{~g}, 4.8 \mathrm{mmol})$ in THF $(80 \mathrm{~mL})$, and ethene was purged through the mixture. 
After $1 \mathrm{~h}$ the mixture was slowly warmed to $20{ }^{\circ} \mathrm{C}$ and stirred for another $6 \mathrm{~h}$. After separation of the organic layer from the amalgam the solvent was removed at reduced pressure, and the residue was taken up with hexane $(30 \mathrm{~mL})$ and filtered through a frit covered with a $3 \mathrm{~cm}$ thick layer of alumina. After solvent removal at reduced pressure complex rac-10 [0.637 g, $1.8 \mathrm{mmol}$, $38 \%$, purity $\left({ }^{1} \mathrm{H} \mathrm{NMR}\right) \geq 95 \%$ ] was obtained as a red oil.

IR: $\tilde{v}=2921(\mathrm{~s}, \mathrm{C}-\mathrm{H}), 2850(\mathrm{~s}, \mathrm{C}-\mathrm{H}), 1447(\mathrm{~m}, \mathrm{C}-\mathrm{H}), 1364$ $(\mathrm{w}, t-\mathrm{Bu}), 1266\left(\mathrm{w}, \mathrm{C}_{2} \mathrm{H}_{4}\right), 1161(\mathrm{~m}), 1013(\mathrm{w}, t-\mathrm{Bu}), 890(\mathrm{w})$, $816(\mathrm{~m}), 793$ (m, Cp), $681(\mathrm{~m}) \mathrm{cm}^{-1} .{ }^{1} \mathrm{H}-\mathrm{NMR}(400 \mathrm{MHz}$, $\left.\mathrm{C}_{6} \mathrm{D}_{6}\right): \delta=1.02\left(\mathrm{~d},{ }^{3} J_{\mathrm{H}, \mathrm{P}}=12.1 \mathrm{~Hz}, 9 \mathrm{H}, \mathrm{CH}_{3}\right), 1.87(\mathrm{~m}, 17 \mathrm{H}$, $\left.7 \mathrm{CH}_{2},=\mathrm{CH}_{2}, \mathrm{PCH}\right), 2.34\left(\mathrm{~m}, 1 \mathrm{H},=\mathrm{CH}_{2}\right), 2.64(\mathrm{~m}, 1 \mathrm{H}$, $\left.=\mathrm{CH}_{2}\right), 3.36\left(\mathrm{~s}, 1 \mathrm{H}, \mathrm{PCH}_{2} \mathrm{CH}_{2} \mathrm{CCHCH}\right), 4.29(\mathrm{~s}, 1 \mathrm{H}$, $\mathrm{PCH}_{2} \mathrm{CH}_{2} \mathrm{CCH}$ ), 5.47 (s, $\left.1 \mathrm{H}, \mathrm{PCH}_{2} \mathrm{CH}_{2} \mathrm{CCHCH}\right), 5.54$ (s, $1 \mathrm{H}$, $\left.\mathrm{PCH}_{2} \mathrm{CH}_{2} \mathrm{CCH}\right)$ ppm. ${ }^{13} \mathrm{C}-\mathrm{NMR}\left(100.6 \mathrm{MHz}, \mathrm{C}_{6} \mathrm{D}_{6}\right): \delta=$ $22.5\left(=\mathrm{CH}_{2}\right), 23.5\left(\mathrm{~d},{ }^{4} J_{\mathrm{C}, \mathrm{P}}=4.6 \mathrm{~Hz}, \mathrm{PCHCH}_{2} \mathrm{CH}_{2} \mathrm{CH}_{2}\right)$, $25.1\left(\mathrm{~d},{ }^{2} J_{\mathrm{C}, \mathrm{P}}=6.9 \mathrm{~Hz}, \mathrm{PCH}_{2} \mathrm{CH}_{2}\right), 27.5\left(\mathrm{~d},{ }^{3} J_{\mathrm{C}, \mathrm{P}}=6.3 \mathrm{~Hz}\right.$, $\left.\mathrm{PCHCH}_{2} \mathrm{CH}_{2}\right), 28.7\left(\mathrm{~d},{ }^{4} J_{\mathrm{C}, \mathrm{P}}=3.4 \mathrm{~Hz}, \mathrm{CH}_{3}\right), 29.4\left(\mathrm{~d},{ }^{2} J_{\mathrm{C}, \mathrm{P}}=\right.$ $5.9 \mathrm{~Hz}, \mathrm{PCHCH}$ ), $31.0\left(\mathrm{~d},{ }^{4} J_{\mathrm{C}, \mathrm{P}}=12.4 \mathrm{~Hz}, \mathrm{PCCH}_{3}\right), 34.8$ $\left(\mathrm{d},{ }^{4} J_{\mathrm{C}, \mathrm{P}}=10.5 \mathrm{~Hz}, \mathrm{PCH}\right), 36.2\left(\mathrm{~d},{ }^{4} J_{\mathrm{C}, \mathrm{P}}=21.2 \mathrm{~Hz}\right.$, $\left.\mathrm{PCH}_{2}\right), 77.2\left(\mathrm{~d},{ }^{4} \mathrm{~J}_{\mathrm{C}, \mathrm{P}}=6.5 \mathrm{~Hz}, \mathrm{PCH}_{2} \mathrm{CH}_{2} \mathrm{CCH}\right), 79.2$ $\left(\mathrm{PCH}_{2} \mathrm{CH}_{2} \mathrm{CCHCH}\right), 80.9 \quad\left(\mathrm{PCH}_{2} \mathrm{CH}_{2} \mathrm{CCHCH}\right), 83.5$ (d, $\left.{ }^{4} J_{\mathrm{C}, \mathrm{P}}=4.6 \mathrm{~Hz}, \mathrm{PCH}_{2} \mathrm{CH}_{2} \mathrm{CCH}\right), 109.3\left(\mathrm{~d},{ }^{3} J_{\mathrm{C}, \mathrm{P}}=6.7 \mathrm{~Hz}\right.$, $\left.\mathrm{PCH}_{2} \mathrm{CH}_{2} \mathrm{CCH}\right)$ ppm. ${ }^{31} \mathrm{P}-\mathrm{NMR}\left(162 \mathrm{MHz}, \mathrm{C}_{6} \mathrm{D}_{6}\right): \delta=$ 93.2 ppm. MS: $m / z(\%)=350$ (10) $\left[\mathrm{M}^{+}\right], 322$ (59) $\left[\mathrm{M}^{+}-\mathrm{CH}_{2}=\mathrm{CH}_{2}\right], 263$ (73) $\left[\mathrm{M}^{+}-\mathrm{CH}_{2}=\mathrm{CH}_{2}-\mathrm{Co}\right], 240$ (30), 183 (69), 136 (55), 83 (61), 57 (100) [t-Bu $\left.{ }^{+}\right], 55$ (73). $\operatorname{HRMS}\left(\mathrm{M}^{+}=\mathrm{C}_{19} \mathrm{H}_{32} \mathrm{CoP}\right)$ calcd 350.1573 , found 350.1571 .

\section{$\left\{\eta^{5}: \eta^{1}[2-(\right.$ Di-tert-butylphosphanyl)ethyl]cyclopentadienyl $\}-$ ( $\eta^{2}$-ethen)-rhodium(I) (rac-11)}

At $-78^{\circ} \mathrm{C}$ butyllithium in hexane $(2.9 \mathrm{~mL}, 4.6 \mathrm{mmol}, 1.6 \mathrm{M})$ was added dropwise to di-tert-butylphosphane $(0.610 \mathrm{~g}, 4.2 \mathrm{mmol})$ in THF (15 mL). After stirring for $2 \mathrm{~h}$ at $21{ }^{\circ} \mathrm{C}$ spiro[2.4]hepta-4,6diene $(4,0.5 \mathrm{~mL}, 5.0 \mathrm{mmol})$ was added, and after stirring for $2 \mathrm{~h}$ at $65{ }^{\circ} \mathrm{C}$ the mixture was cooled to $-78{ }^{\circ} \mathrm{C}$, and $\left[\mathrm{Rh}_{2}\left(\mathrm{C}_{2} \mathrm{H}_{4}\right)_{4} \mathrm{Cl}_{2}\right]$ $(0.982 \mathrm{~g}, 2.5 \mathrm{mmol})$ was added. The mixture was slowly warmed to $20{ }^{\circ} \mathrm{C}$ and stirred for another $2 \mathrm{~h}$. After solvent removal at reduced pressure the residue was taken up with pentane $(15 \mathrm{~mL})$ and filtered through a frit covered with a $3 \mathrm{~cm}$ thick layer of Celite. The solvent was removed at reduced pressure affording $\mathrm{rac}-\mathbf{1 1}$ $\left[1.120 \mathrm{~g}, 3.0 \mathrm{mmol}, 72 \%\right.$, purity $\left.\geq 95 \% \quad\left({ }^{1} \mathrm{H} \quad \mathrm{NMR}\right)\right]$ as a brown oil.

IR: $\tilde{v}=2958$ (m, C-H), 2896 (m, C-H), 1471 (m, C-H), 1386 (w), 1366 (m, $t$-Bu), 1167 (s), 1019 (m, t-Bu), $928(\mathrm{w}), 809(\mathrm{~m})$, $772(\mathrm{~m}), 661(\mathrm{w}) \mathrm{cm}^{-1} \cdot{ }^{1} \mathrm{H}-\mathrm{NMR}\left(400 \mathrm{MHz}, \mathrm{C}_{6} \mathrm{D}_{6}\right): \delta=1.06$ $\left(\mathrm{d}, 18 \mathrm{H},{ }^{3} J_{\mathrm{H}, \mathrm{P}}=12.2 \mathrm{~Hz}, \mathrm{CH}_{3}\right), 1.88\left(\mathrm{~m}, 2 \mathrm{H}, \mathrm{PCH}_{2} \mathrm{CH}_{2}\right), 2.02$ $\left(\mathrm{m}, 2 \mathrm{H}, \mathrm{PCH}_{2}\right), 2.40\left(\mathrm{~m}, 2 \mathrm{H}, \mathrm{CH}_{2}=\mathrm{CH}_{2}\right), 2.78(\mathrm{~m}, 2 \mathrm{H}$, $\left.\mathrm{CH}_{2}=\mathrm{CH}_{2}\right), 4.72\left(\mathrm{~m}, 2 \mathrm{H}, \mathrm{PCH}_{2} \mathrm{CH}_{2} \mathrm{CCHCH}\right), 5.51(\mathrm{~m}, 2 \mathrm{H}$, $\left.\mathrm{PCH}_{2} \mathrm{CH}_{2} \mathrm{CCH}\right)$ ppm. ${ }^{13} \mathrm{C}-\mathrm{NMR}\left(100.6 \mathrm{MHz}, \mathrm{C}_{6} \mathrm{D}_{6}\right): \delta=$ $25.5\left(\mathrm{dd},{ }^{2} J_{\mathrm{C}, \mathrm{P}}=15.1 \mathrm{~Hz},{ }^{3} J_{\mathrm{C}, \mathrm{Rh}}=2.4 \mathrm{~Hz}, \mathrm{PCH}_{2} \mathrm{CH}_{2}\right), 30.1$ $\left(\mathrm{d},{ }^{2} J_{\mathrm{C}, \mathrm{P}}=4.9 \mathrm{~Hz}, \mathrm{CH}_{3}\right), 35.5\left(\mathrm{dd},{ }^{1} J_{\mathrm{C}, \mathrm{P}}=9.5 \mathrm{~Hz},{ }^{2} J_{\mathrm{C}, \mathrm{Rh}}=\right.$ $\left.2.4 \mathrm{~Hz}, \mathrm{PCCH}_{3}\right), 37.9\left(\mathrm{dd},{ }^{2} J_{\mathrm{C}, \mathrm{P}}=13.4 \mathrm{~Hz},{ }^{1} J_{\mathrm{C}, \mathrm{Rh}}=2.1 \mathrm{~Hz}\right.$, $\left.\mathrm{H}_{2} \mathrm{C}=\mathrm{CH}_{2}\right), 42.4\left(\mathrm{~d},{ }^{1} J_{\mathrm{C}, \mathrm{P}}=19.5 \mathrm{~Hz}, \mathrm{PCH}_{2}\right), 82.9(\mathrm{dd}$, $J_{\mathrm{C}, \mathrm{P}}=4.0 \mathrm{~Hz},{ }^{1} J_{\mathrm{C}, \mathrm{Rh}}=3.0 \mathrm{~Hz}, \mathrm{PCH}_{2} \mathrm{CH}_{2} \mathrm{CCHCH}$ or $\left.\mathrm{PCH}_{2} \mathrm{CH}_{2} \mathrm{CCH}\right), 86.2\left(\mathrm{dd}, J_{\mathrm{C}, \mathrm{P}}=8.4 \mathrm{~Hz},{ }^{1} J_{\mathrm{C}, \mathrm{Rh}}=2.8 \mathrm{~Hz}\right.$, $\mathrm{PCH}_{2} \mathrm{CH}_{2} \mathrm{CCHCH}$ or $\left.\mathrm{PCH}_{2} \mathrm{CH}_{2} \mathrm{CCH}\right), 113.6\left(\mathrm{dd}, J_{\mathrm{C}, \mathrm{P}}=6.4 \mathrm{~Hz}\right.$, $\left.{ }^{1} J_{\mathrm{C}, \mathrm{Rh}}=4.2 \mathrm{~Hz}, \mathrm{PCH}_{2} \mathrm{CH}_{2} C\right)$ ppm. ${ }^{31} \mathrm{P}-\mathrm{NMR}(162 \mathrm{MHz}$,
$\left.\mathrm{C}_{6} \mathrm{D}_{6}\right): \delta=99.3\left(\mathrm{~d},{ }^{1} J_{\mathrm{P}, \mathrm{Rh}}=211.7 \mathrm{~Hz}\right) \mathrm{ppm}$. MS: $m / z(\%)=$ 368 (18) $\left[\mathrm{M}^{+}\right], 340$ (60) $\left[\mathrm{M}^{+}-\mathrm{CH}_{2}=\mathrm{CH}_{2}\right], 284$ (100), 227 (45), 224 (56), 180 (30), 57 (76) $\left[t-\mathrm{Bu}^{+}\right]$. HRMS $\left(\mathrm{M}^{+}=\mathrm{C}_{17} \mathrm{H}_{30} \mathrm{RhP}\right)$ calcd 368.1137 , found 368.1140 .

\section{$\left\{\eta^{5}: \eta^{1}[2-(\right.$ tert-Butylphenylphosphanyl)ethyl]cyclopentadienyl $\}$ - ( $\eta^{2}$-ethen)-rhodium(I) (rac-12)}

At $-78{ }^{\circ} \mathrm{C}$ butyllithium in hexane $(6.4 \mathrm{~mL}, 10.2 \mathrm{mmol}, 1.6 \mathrm{M})$ was added dropwise to di-tert-butylphosphane $(1.438 \mathrm{~g}, 8.7 \mathrm{mmol})$ in THF (30 mL). After stirring for $2 \mathrm{~h}$ at $21{ }^{\circ} \mathrm{C}$ spiro[2.4]hepta-4,6diene $(4,0.9 \mathrm{~mL}, 9.0 \mathrm{mmol})$ was added, and after stirring for $2 \mathrm{~h}$ at $65{ }^{\circ} \mathrm{C}$ the mixture was cooled to $-78{ }^{\circ} \mathrm{C}$, and $\left[\mathrm{Rh}_{2}\left(\mathrm{C}_{2} \mathrm{H}_{4}\right)_{4} \mathrm{Cl}_{2}\right]$ $(2.020 \mathrm{~g}, 5.2 \mathrm{mmol})$ was added. The mixture was slowly warmed to $20{ }^{\circ} \mathrm{C}$ and stirred for another $2 \mathrm{~h}$. After solvent removal at reduced pressure the residue was taken up with pentane $(15 \mathrm{~mL})$ and filtered through a frit covered with a $3 \mathrm{~cm}$ thick layer of Celite. The solvent was removed at reduced pressure and the residue was taken up with $3 \mathrm{~mL}$ of toluene. As attempts to crystallize the product at $-25{ }^{\circ} \mathrm{C}$ failed, the solvent was removed at reduced pressure affording rac-12 $(2.850 \mathrm{~g}, 7.7 \mathrm{mmol}, 85 \%)$ as a brown oil, purity $\geq 95 \%\left({ }^{1} \mathrm{H} \mathrm{NMR}\right)$.

IR: $\tilde{v}=3045\left(\mathrm{w}, \mathrm{C}_{2} \mathrm{H}_{4}\right), 2941(\mathrm{~m}, \mathrm{C}-\mathrm{H}), 2861(\mathrm{w}, \mathrm{C}-\mathrm{H}), 1474$ (w, C-H), 1433 (m, P-Ph), $1416(\mathrm{w}), 1390(\mathrm{w}), 1362$ (m, $t$-Bu), 1308 (m), 1266 (w, kompl. $\mathrm{C}_{2} \mathrm{H}_{4}$ ), 1167 (w), 1095 (s), 1028 (m, t-Bu), 1013 (w), 929 (w), 841 (m, Cp), 809 (s, Cp), 772 (m), 744 (m), 697 (s), $668(\mathrm{~m}), 620$ (s) $\mathrm{cm}^{-1} .{ }^{1} \mathrm{H}-\mathrm{NMR}(400 \mathrm{MHz}$, $\left.\mathrm{C}_{6} \mathrm{D}_{6}\right): \delta=0.87\left(\mathrm{~d},{ }^{3} J_{\mathrm{H}, \mathrm{P}}=13.6 \mathrm{~Hz}, 9 \mathrm{H}, \mathrm{CH}_{3}\right), 1.59(\mathrm{~m}, 1 \mathrm{H}$, $\left.\mathrm{PCH}_{2} \mathrm{CH}_{2}\right), 2.03\left(\mathrm{dddd},{ }^{2} J_{\mathrm{H}, \mathrm{P}}=45.6, J_{\mathrm{H}, \mathrm{H}}=13.5,6.6\right.$, $\left.2.4 \mathrm{~Hz}, 1 \mathrm{H}, \mathrm{PCH}_{2} \mathrm{CH}_{2}\right), 2.24\left(\mathrm{~m}, 1 \mathrm{H}, \mathrm{PCH}_{2}\right), 2.31(\mathrm{~m}, 1 \mathrm{H}$, $\left.=\mathrm{CH}_{2}\right), 2.50\left(\mathrm{~m}, 1 \mathrm{H},=\mathrm{CH}_{2}\right), 2.54\left(\mathrm{~m}, 1 \mathrm{H}, \mathrm{PCH}_{2}\right), 2.93$ $\left(\mathrm{m}, 1 \mathrm{H},=\mathrm{CH}_{2}\right), 3.11\left(\mathrm{~m}, 1 \mathrm{H},=\mathrm{CH}_{2}\right), 4.70\left(\mathrm{~d},{ }^{4} J_{\mathrm{H}, \mathrm{P}}=\right.$ $\left.2.7 \mathrm{~Hz}, 1 \mathrm{H}, \mathrm{PCH}_{2} \mathrm{CH}_{2} \mathrm{CCH}\right), 4.99\left(\mathrm{~d},{ }^{3} J_{\mathrm{H}, \mathrm{P}}=2.0 \mathrm{~Hz}\right.$, $\left.1 \mathrm{H}, \mathrm{PCH}_{2} \mathrm{CH}_{2} \mathrm{CCH}\right), 5.23\left(\mathrm{~d},{ }^{5} \mathrm{~J}_{\mathrm{H}, \mathrm{P}}=1.0 \mathrm{~Hz}, 1 \mathrm{H}, \mathrm{PCH}_{2} \mathrm{CH}_{2}-\right.$ $\mathrm{CCHCH}), 5.60\left(\mathrm{~d},{ }^{5} J_{\mathrm{H}, \mathrm{P}}=1.3 \mathrm{~Hz}, 1 \mathrm{H}, \mathrm{PCH}_{2} \mathrm{CH}_{2} \mathrm{CCHCH}\right)$, $7.17(\mathrm{~m}, 3 \mathrm{H}, o-\mathrm{CH}, p-\mathrm{CH}), 7.89(\mathrm{~m}, 2 \mathrm{H}, m-\mathrm{CH}) \mathrm{ppm} .{ }^{13} \mathrm{C}-\mathrm{NMR}$ $\left(100.6 \mathrm{MHz}, \mathrm{C}_{6} \mathrm{D}_{6}\right): \delta=22.7\left(\mathrm{~d},{ }^{2} J_{\mathrm{C}, \mathrm{P}}=2.6 \mathrm{~Hz}, \mathrm{PCH}_{2} \mathrm{CH}_{2}\right)$, $25.9\left(\mathrm{dd},{ }^{2} J_{\mathrm{C}, \mathrm{P}}=15.1 \mathrm{~Hz},{ }^{1} J_{\mathrm{C}, \mathrm{Rh}}=2.4 \mathrm{~Hz},=\mathrm{CH}_{2}\right), 26.5$ $\left(\mathrm{d},{ }^{2} J_{\mathrm{C}, \mathrm{P}}=4.7 \mathrm{~Hz}, \mathrm{CH}_{3}\right), 32.0\left(\mathrm{~d},{ }^{1} J_{\mathrm{C}, \mathrm{P}}=16.7 \mathrm{~Hz},{ }^{2} J_{\mathrm{C}, \mathrm{Rh}}=\right.$ $\left.2.7 \mathrm{~Hz}, \mathrm{PCCH}_{3}\right), 42.2\left(\mathrm{~d},{ }^{1} J_{\mathrm{C}, \mathrm{P}}=24.5 \mathrm{~Hz}, \mathrm{PCH}_{2}\right), 80.8(\mathrm{dd}$, $\left.{ }^{5} J_{\mathrm{C}, \mathrm{P}}=4.2 \mathrm{~Hz},{ }^{1} J_{\mathrm{C}, \mathrm{Rh}}=2.6 \mathrm{~Hz}, \mathrm{PCH}_{2} \mathrm{CH}_{2} \mathrm{CCHCH}\right), 84.8$ $\left(\mathrm{dd},{ }^{5} J_{\mathrm{C}, \mathrm{P}}=3.8 \mathrm{~Hz},{ }^{1} J_{\mathrm{C}, \mathrm{Rh}}=3.0 \mathrm{~Hz}, \mathrm{PCH}_{2} \mathrm{CH}_{2} \mathrm{CCHCH}\right)$, $85.3\left(\mathrm{dd},{ }^{4} J_{\mathrm{C}, \mathrm{P}}=8.8 \mathrm{~Hz},{ }^{1} J_{\mathrm{C}, \mathrm{Rh}}=2.8 \mathrm{~Hz}, \mathrm{PCH}_{2} \mathrm{CH}_{2} \mathrm{CCH}\right)$, $88.9\left(\mathrm{dd},{ }^{4} J_{\mathrm{C}, \mathrm{P}}=8.4 \mathrm{~Hz},{ }^{1} J_{\mathrm{C}, \mathrm{Rh}}=2.7 \mathrm{~Hz}, \mathrm{PCH}_{2} \mathrm{CH}_{2} \mathrm{CCH}\right)$, $112.0\left(\mathrm{dd},{ }^{3} J_{\mathrm{C}, \mathrm{P}}=5.5 \mathrm{~Hz},{ }^{1} J_{\mathrm{C}, \mathrm{Rh}}=4.2 \mathrm{~Hz}, \mathrm{PCH}_{2} \mathrm{CH}_{2} C\right)$, $127.5\left(\mathrm{~d},{ }^{2} J_{\mathrm{C}, \mathrm{P}}=8.8 \mathrm{~Hz}, o-\mathrm{CH}\right), 129.4\left(\mathrm{~d},{ }^{4} J_{\mathrm{C}, \mathrm{P}}=2.3 \mathrm{~Hz}\right.$, $p-\mathrm{CH}), 134.1\left(\mathrm{~d},{ }^{3} J_{\mathrm{C}, \mathrm{P}}=10.5 \mathrm{~Hz}, m-\mathrm{CH}\right), 136.3\left(\mathrm{dd},{ }^{1} J_{\mathrm{C}, \mathrm{P}}=\right.$ $\left.25.1 \mathrm{~Hz},{ }^{2} J_{\mathrm{C}, \mathrm{Rh}}=1.5 \mathrm{~Hz}, \mathrm{PCCH}\right) \mathrm{ppm} .{ }^{31} \mathrm{P}-\mathrm{NMR}(162 \mathrm{MHz}$, $\left.\mathrm{C}_{6} \mathrm{D}_{6}\right): \delta=79.7\left(\mathrm{~d},{ }^{1} J_{\mathrm{P}, \mathrm{Rh}}=215 \mathrm{~Hz}\right)$ ppm. MS: $m / z(\%)=$ 388 (47) $\left[\mathrm{M}^{+}\right], 360$ (99) $\left[\mathrm{M}^{+}-\mathrm{CH}_{2}=\mathrm{CH}_{2}\right], 304$ (100) $\left[\mathrm{M}^{+}-\mathrm{C}_{4} \mathrm{H}_{8}\right], 223$ (34), 180 (50), 57 (39) $\left[t-\mathrm{Bu}^{+}\right]$. HRMS $\left(\mathrm{M}^{+}=\mathrm{C}_{19} \mathrm{H}_{26} \mathrm{RhP}\right)$ calcd 388.0827, found 388.0828. $T_{\mathrm{c}}=330 \mathrm{~K}$ (d $\mathrm{d}_{6}$-benzene $) . \Delta G^{\ddagger}=62.98 \mathrm{~kJ} \mathrm{~mol}^{-1}$. $T_{\mathrm{c}}=325 \mathrm{~K}$ (d8-toluene), $\Delta G^{\ddagger}=62.5 \mathrm{~kJ} \mathrm{~mol}^{-1}$.

\section{$\left\{\eta^{5}: \eta^{1}[2-(\right.$ tert-Butylcyclohexylphosphanyl)ethyl]- cyclopentadienyl $\}-\left(\eta^{2}\right.$-ethen)rhodium(I) (rac-13)}

At $-78{ }^{\circ} \mathrm{C}$ butyllithium in hexane $(2.9 \mathrm{~mL}, 4.6 \mathrm{mmol}$, $1.6 \mathrm{M})$ was added dropwise to tert-butylcyclohexylphosphane 
$(0.726 \mathrm{~g}, 4.2 \mathrm{mmol})$ in THF $(15 \mathrm{~mL})$. After stirring for $2 \mathrm{~h}$ at $20{ }^{\circ} \mathrm{C}$ spiro[2.4]hepta-4,6-diene $(4,0.9 \mathrm{~mL}, 9.0 \mathrm{mmol})$ was added, and after stirring for $2 \mathrm{~h}$ at $65{ }^{\circ} \mathrm{C}$ the mixture was cooled to $-78{ }^{\circ} \mathrm{C}$, and $\left[\mathrm{Rh}_{2}\left(\mathrm{C}_{2} \mathrm{H}_{4}\right)_{4} \mathrm{Cl}_{2}\right](0.982 \mathrm{~g}, 2.5 \mathrm{mmol})$ was added. The mixture was slowly warmed to $20{ }^{\circ} \mathrm{C}$ and stirred for another $2 \mathrm{~h}$. After solvent removal at reduced pressure the residue was taken up with pentane $(15 \mathrm{~mL})$ and filtered through a frit covered with a $3 \mathrm{~cm}$ thick layer of Celite. The solvent was removed at reduced pressure affording $\mathbf{r a c}-\mathbf{1 3}$ (0.910 g, $2.3 \mathrm{mmol}, 55 \%)$ as a brown oil.

IR: $\tilde{v}=2920$ (s, C-H), 2849 (s, C-H), $1944(\mathrm{~m}), 1782(\mathrm{w})$, 1447 (m, C-H), 1415 (w), 1391 (w), 1363 (m, t-Bu), 1268 (w, $\mathrm{C}_{2} \mathrm{H}_{4}$ ), $1184(\mathrm{~m}), 1168$ (s), $1026(\mathrm{~m}), 1012$ (m, $t$-Bu), 930 (m), 887 (w), 850 (w), 810 (m), 769 (s, Cp), 730 (m) cm ${ }^{-1} .{ }^{1} \mathrm{H}-\mathrm{NMR}$ $\left(400 \mathrm{MHz}, \mathrm{C}_{6} \mathrm{D}_{6}\right): \delta=0.94\left(\mathrm{~d},{ }^{3} J_{\mathrm{H}, \mathrm{P}}=12.1 \mathrm{~Hz}, 9 \mathrm{H}, \mathrm{CH}_{3}\right)$, $1.81\left(\mathrm{~m}, 17 \mathrm{H}, 7 \mathrm{CH} 2,=\mathrm{CH}_{2}\right), 2.86\left(\mathrm{~m}, 2 \mathrm{H},=\mathrm{CH}_{2}\right), 4.71$ $\left(\mathrm{d},{ }^{4} J_{\mathrm{H}, \mathrm{P}}=2.4 \mathrm{~Hz}, 1 \mathrm{H}, \mathrm{PCH}_{2} \mathrm{CH}_{2} \mathrm{CCHCH}\right), 5.00(\mathrm{~s}, 1 \mathrm{H}$, $\mathrm{PCH}_{2} \mathrm{CH}_{2} \mathrm{CCH}$ ), 5.54 (d, ${ }^{5} \mathrm{~J}_{\mathrm{H}, \mathrm{P}}=1.0 \mathrm{~Hz}, 1 \mathrm{H}, \mathrm{PCH}_{2} \mathrm{CH}_{2} \mathrm{CCH}$ ), $5.60\left(\mathrm{~d},{ }^{5} J_{\mathrm{H}, \mathrm{P}}=1.4 \mathrm{~Hz}, 1 \mathrm{H}, \mathrm{PCH}_{2} \mathrm{CH}_{2} \mathrm{CCHCH}\right) \mathrm{ppm}$. ${ }^{13} \mathrm{C}-\mathrm{NMR}\left(100.6 \mathrm{MHz}, \mathrm{C}_{6} \mathrm{D}_{6}\right): \delta=25.3\left(\mathrm{~d},{ }^{2} J_{\mathrm{C}, \mathrm{P}}=2.8 \mathrm{~Hz}\right.$, $\left.\mathrm{PCH}_{2} \mathrm{CH}_{2}\right), 26.6\left(\mathrm{~d},{ }^{4} \mathrm{~J}_{\mathrm{C}, \mathrm{P}}=1.3 \mathrm{~Hz}, \mathrm{PCHCH}_{2} \mathrm{CH}_{2} \mathrm{CH}_{2}\right), 27.6$ $\left(\mathrm{d},{ }^{3} J_{\mathrm{C}, \mathrm{P}}=8.0 \mathrm{~Hz}, \mathrm{PCHCH}_{2} \mathrm{CH}_{2}\right), 28.0\left(\mathrm{~d},{ }^{2} J_{\mathrm{C}, \mathrm{P}}=13.2 \mathrm{~Hz}\right.$, $\left.=\mathrm{CH}_{2}\right), 28.4\left(\mathrm{~d},{ }^{2} J_{\mathrm{C}, \mathrm{P}}=4.2 \mathrm{~Hz}, \mathrm{CH}_{3}\right), 29.3\left(\mathrm{~d},{ }^{2} J_{\mathrm{C}, \mathrm{P}}=3.2\right.$ $\mathrm{Hz}, \mathrm{PCHCH}$ ), $32.1\left(\mathrm{dd},{ }^{1} J_{\mathrm{C}, \mathrm{P}}=15.5 \mathrm{~Hz},{ }^{2} J_{\mathrm{C}, \mathrm{Rh}}=2.3 \mathrm{~Hz}\right.$, $\left.\mathrm{PCCH}_{3}\right), 35.8\left(\mathrm{dd},{ }^{1} J_{\mathrm{C}, \mathrm{P}}=13.3 \mathrm{~Hz},{ }^{2} J_{\mathrm{C}, \mathrm{Rh}}=2.0 \mathrm{~Hz}, \mathrm{PCH}\right)$, $41.1\left(\mathrm{~d},{ }^{1} J_{\mathrm{C}, \mathrm{P}}=21.0 \mathrm{~Hz}, \mathrm{PCH}_{2}\right), 81.7\left(\mathrm{dd},{ }^{5} J_{\mathrm{C}, \mathrm{P}}=4.1 \mathrm{~Hz}\right.$, $\left.{ }^{1} J_{\mathrm{C}, \mathrm{Rh}}=2.5 \mathrm{~Hz}, \mathrm{PCH}_{2} \mathrm{CH}_{2} \mathrm{CCHCH}\right), 82.6\left(\mathrm{dd},{ }^{5} J_{\mathrm{C}, \mathrm{P}}=4.0\right.$ $\left.\mathrm{Hz},{ }^{1} J_{\mathrm{C}, \mathrm{Rh}}=3.0 \mathrm{~Hz}, \mathrm{PCH}_{2} \mathrm{CH}_{2} \mathrm{CCHCH}\right), 85.2\left(\mathrm{dd},{ }^{4} J_{\mathrm{C}, \mathrm{P}}=\right.$ $\left.9.2 \mathrm{~Hz},{ }^{1} J_{\mathrm{C}, \mathrm{Rh}}=2.6 \mathrm{~Hz}, \mathrm{PCH}_{2} \mathrm{CH}_{2} \mathrm{CCH}\right), 88.1\left(\mathrm{dd},{ }^{4} J_{\mathrm{C}, \mathrm{P}}=\right.$ $\left.7.4 \mathrm{~Hz},{ }^{1} J_{\mathrm{C}, \mathrm{Rh}}=2.8 \mathrm{~Hz}, \mathrm{PCH}_{2} \mathrm{CH}_{2} \mathrm{CCHCH}\right), 113.1(\mathrm{dd}$, $\left.{ }^{3} J_{\mathrm{C}, \mathrm{P}}=6.3 \mathrm{~Hz},{ }^{1} J_{\mathrm{C}, \mathrm{Rh}}=4.2 \mathrm{~Hz}, \mathrm{PCH}_{2} \mathrm{CH}_{2} C\right)$ ppm. ${ }^{31} \mathrm{P}-\mathrm{NMR}\left(162 \mathrm{MHz}, \mathrm{C}_{6} \mathrm{D}_{6}\right): \delta=91.5\left(\mathrm{~d},{ }^{1} J_{\mathrm{P}, \mathrm{Rh}}=\right.$ $209.6 \mathrm{~Hz}) \mathrm{ppm}$. MS: $m / z(\%)=394(12)\left[\mathrm{M}^{+}\right], 366(41)$ $\left[\mathrm{M}^{+}-\mathrm{CH}_{2}=\mathrm{CH}_{2}\right], 284$ (34), 224 (33), 166 (24), 82 (100) $\left[\mathrm{C}_{6} \mathrm{H}_{10}{ }^{+}\right], 57$ (57) $\left[t-\mathrm{Bu}^{+}\right]$. HRMS $\left(\mathrm{M}^{+}=\mathrm{C}_{19} \mathrm{H}_{32} \mathrm{RhP}\right)$ calcd 394.1296, found 394.1298.

\section{$\left\{\eta^{5}: \eta^{1}[2-(\right.$ tert-Butylphenylphosphanyl)ethyl]- cyclopentadienyl\}iodomethyl-rhodium(II) (rac-14 and rac-15)}

At $22{ }^{\circ} \mathrm{C}$ iodomethane $(0.6 \mathrm{~mL}, 9.0 \mathrm{mmol})$ was added to rhodium(I) complex rac-11 $(767 \mathrm{mg}, 1.9 \mathrm{mmol})$ in THF $(120 \mathrm{~mL})$. After stirring at $22{ }^{\circ} \mathrm{C}$ for $24 \mathrm{~h}$ the solvent was removed at reduced pressure, and the residue was taken up with dichloromethane $(20 \mathrm{~mL})$. The mixture was filtered through a frit covered with a $3 \mathrm{~cm}$ thick layer of Celite. The filtrate was concentrated and the product was crystallized from dichloromethane/hexane at $-25{ }^{\circ} \mathrm{C}$. The product was obtained as a diastereomeric mixture of $r a c-14$ and $r a c-15$ (100: 0.7, ${ }^{1} \mathrm{H}$ NMR) as a red solid (593 mg, $1.2 \mathrm{mmol}, 60 \%$ ).

IR $(95 \%$ de): $\tilde{v}=2958(\mathrm{~m}, \mathrm{C}-\mathrm{H}), 2893(\mathrm{~m}, \mathrm{C}-\mathrm{H}), 1460(\mathrm{~m}$, C-H), 1432 (m, P-Ph), 1260 (m), 1168 (w), 1125 (m), 1096 (s), 1015 (s, t-Bu), 840 (m, Cp), 797 (s, Cp), 746 (m), 698 (s) cm c. $^{-1}$. rac-14: ${ }^{1} \mathrm{H}-\mathrm{NMR}\left(500 \mathrm{MHz}, \mathrm{CDCl}_{3}\right): \delta=1.06(\mathrm{dd}, J=5.4$, $\left.2.3 \mathrm{~Hz}, 3 \mathrm{H}, \mathrm{RhCH}_{3}\right), 1.15\left(\mathrm{~d},{ }^{3} J_{\mathrm{H}, \mathrm{P}}=14.8 \mathrm{~Hz}, 9 \mathrm{H}, \mathrm{CCH}_{3}\right)$, $2.17\left(\mathrm{~d},{ }^{3} J_{\mathrm{H}, \mathrm{H}}=6.9 \mathrm{~Hz},{ }^{3} J_{\mathrm{H}, \mathrm{H}}=6.0 \mathrm{~Hz},{ }^{3} J_{\mathrm{H}, \mathrm{P}}=34.7 \mathrm{~Hz}\right.$, $\left.{ }^{2} J_{\mathrm{H}, \mathrm{H}}=-14.1 \mathrm{~Hz}, 1 \mathrm{H}, \mathrm{PCH}_{2} \mathrm{CH}_{2}\right), 2.32\left(\mathrm{~d}, 1 \mathrm{H},{ }^{3} J_{\mathrm{H}, \mathrm{H}}=6.0\right.$ $\mathrm{Hz},{ }^{3} J_{\mathrm{H}, \mathrm{H}}=9.8 \mathrm{~Hz},{ }^{3} J_{\mathrm{H}, \mathrm{P}}=20.6 \mathrm{~Hz},{ }^{2} J_{\mathrm{H}, \mathrm{H}}=-14.1 \mathrm{~Hz}$, $\left.\mathrm{PCH}_{2} \mathrm{CH}_{2}\right), 2.84\left(\mathrm{~d},{ }^{3} J_{\mathrm{H}, \mathrm{H}}=9.8 \mathrm{~Hz},{ }^{3} J_{\mathrm{H}, \mathrm{H}}=6.9 \mathrm{~Hz},{ }^{2} J_{\mathrm{H}, \mathrm{P}}=\right.$
$9.8 \mathrm{~Hz},{ }^{3} J_{\mathrm{H}, \mathrm{H}}=-14.2 \mathrm{~Hz},{ }^{3} J_{\mathrm{H}, \mathrm{Rh}}=0.6 \mathrm{~Hz}, 1 \mathrm{H}, \mathrm{PCH}_{2}$ ), $3.23\left(\mathrm{~d},{ }^{3} J_{\mathrm{H}, \mathrm{H}}=6.0 \mathrm{~Hz},{ }^{3} J_{\mathrm{H}, \mathrm{H}}=6.0 \mathrm{~Hz},{ }^{2} J_{\mathrm{H}, \mathrm{P}}=9.0 \mathrm{~Hz}\right.$, $\left.{ }^{3} J_{\mathrm{H}, \mathrm{H}}=-14.2 \mathrm{~Hz},{ }^{3} J_{\mathrm{H}, \mathrm{Rh}}=1.2 \mathrm{~Hz}, 1 \mathrm{H}, \mathrm{PCH}_{2}\right), 4.50(\mathrm{~m}, 1 \mathrm{H}$, $\left.\mathrm{PCH}_{2} \mathrm{CH}_{2} \mathrm{CCH}\right), 5.15\left(\mathrm{~m}, 1 \mathrm{H}, \mathrm{PCH}_{2} \mathrm{CH}_{2} \mathrm{CCHCH}\right), 5.40(\mathrm{~m}$, $\left.1 \mathrm{H}, \mathrm{PCH}_{2} \mathrm{CH}_{2} \mathrm{CCH}\right), 5.89\left(\mathrm{~m}, 1 \mathrm{H}, \mathrm{PCH}_{2} \mathrm{CH}_{2} \mathrm{CCHCH}\right)$, $7.28(\mathrm{~m}, 5 \mathrm{H}, \mathrm{Ph}-\mathrm{H})$ ppm. ${ }^{13} \mathrm{C}-\mathrm{NMR}\left(100.6 \mathrm{MHz}, \mathrm{CDCl}_{3}\right)$ : $\delta=-13.5\left(\mathrm{dd},{ }^{2} J_{\mathrm{C}, \mathrm{P}}=20.9 \mathrm{~Hz},{ }^{1} J_{\mathrm{C}, \mathrm{Rh}}=10.4 \mathrm{~Hz}, \mathrm{RhCH}_{3}\right)$, $23.3\left(\mathrm{~d},{ }^{2} J_{\mathrm{C}, \mathrm{P}}=1.3 \mathrm{~Hz}, \mathrm{PCH}_{2} \mathrm{CH}_{2}\right), 27.9\left(\mathrm{~d},{ }^{2} J_{\mathrm{C}, \mathrm{P}}=3.4 \mathrm{~Hz}\right.$, $\left.\mathrm{CCH}_{3}\right), 35.0\left(\mathrm{~d},{ }^{1} J_{\mathrm{C}, \mathrm{P}}=19.1 \mathrm{~Hz},{ }^{2} J_{\mathrm{C}, \mathrm{Rh}}=0.5 \mathrm{~Hz}, \mathrm{CCH}_{3}\right)$, $42.5\left(\mathrm{~d},{ }^{1} J_{\mathrm{C}, \mathrm{P}}=24.9 \mathrm{~Hz}, \mathrm{PCH}_{2}\right), 81.7\left(\mathrm{dd}, J_{\mathrm{C}, \mathrm{P}}=6.9 \mathrm{~Hz},{ }^{1} J_{\mathrm{C}, \mathrm{Rh}}=\right.$ $\left.3.1 \mathrm{~Hz}, \quad \mathrm{PCH}_{2} \mathrm{CH}_{2} \mathrm{CCH}\right), 84.9\left(\mathrm{dd}, \quad J_{\mathrm{C}, \mathrm{P}}=3.2 \mathrm{~Hz}\right.$, $\left.\mathrm{PCH}_{2} \mathrm{CH}_{2} \mathrm{CCH}\right), 87.2\left(\mathrm{dd}, J_{\mathrm{C}, \mathrm{P}}=11.3 \mathrm{~Hz}, J_{\mathrm{C}, \mathrm{Rh}}=3.6 \mathrm{~Hz}\right.$, $\left.\mathrm{PCH}_{2} \mathrm{CH}_{2} \mathrm{CCHCH}\right), 102.7\left(\mathrm{dd}, J_{\mathrm{C}, \mathrm{P}}=5.7 \mathrm{~Hz}, J_{\mathrm{C}, \mathrm{Rh}}=2.3 \mathrm{~Hz}\right.$, $\left.\mathrm{PCH}_{2} \mathrm{CH}_{2} \mathrm{CCHCH}\right), 118.3\left(\mathrm{dd},{ }^{3} J_{\mathrm{C}, \mathrm{P}}=7.7 \mathrm{~Hz}, J_{\mathrm{C}, \mathrm{Rh}}=\right.$ $\left.5.0 \mathrm{~Hz}, \mathrm{PCH}_{2} \mathrm{CH}_{2} C\right), 127.8\left(\mathrm{~d},{ }^{2} J_{\mathrm{C}, \mathrm{P}}=9.0 \mathrm{~Hz}, o-\mathrm{CH}\right), 130.0$ $\left(\mathrm{d},{ }^{4} J_{\mathrm{C}, \mathrm{P}}=2.4 \mathrm{~Hz}, p-\mathrm{CH}\right), 132.9\left(\mathrm{~d},{ }^{3} J_{\mathrm{C}, \mathrm{P}}=7.2 \mathrm{~Hz}, m-\mathrm{CH}\right)$, $133.9\left(\mathrm{dd},{ }^{1} J_{\mathrm{C}, \mathrm{P}}=8.4 \mathrm{~Hz}, \mathrm{PCCH}\right) \mathrm{ppm} .{ }^{31} \mathrm{P}-\mathrm{NMR}(162 \mathrm{MHz}$, $\left.\mathrm{C}_{6} \mathrm{D}_{6}\right): \delta=75.7\left(\mathrm{~d},{ }^{1} J_{\mathrm{P}, \mathrm{Rh}}=167 \mathrm{~Hz}\right) \mathrm{ppm}$. MS $(95 \% \mathrm{de})$ : $m / z(\%)=501(14)\left[\mathrm{M}^{+}\right], 486(61)\left[\mathrm{M}^{+}-\mathrm{CH}_{3}\right], 360(61)$ $\left[\mathrm{M}^{+}-\mathrm{CH}_{3}-\mathrm{I}\right], 302$ (100), 180 (40), 68 (24), 57 (40) $\left[t-\mathrm{Bu}^{+}\right]$. $\operatorname{HRMS}\left(\mathrm{M}^{+}=\mathrm{C}_{18} \mathrm{H}_{25} \mathrm{PRhI}\right)$ calcd 501.9793, found 501.9791.

Crystal structure analysis of $\mathrm{rac}-\mathbf{1 4}:^{45}$ empirical formula $\mathrm{C}_{18} \mathrm{H}_{25}$ IPRh, molecular weight 502.16, crystal system orthorhombic, space group Pbca, $a=21.093(9) \AA$, $b=16.109(5) \AA, c=21.823(6) \AA, \alpha=90.00^{\circ}, \beta=90.00^{\circ}$, $\gamma=90.00^{\circ}, V=7415(4) \AA^{3}, Z=16, d_{\text {calcd }}=1.799 \mathrm{~g} \mathrm{~cm}^{-1}$, $F(000)=3936.0, \mu=2.665 \mathrm{~mm}^{-1}$, Stoe IPDS diffractometer, $T=297 \mathrm{~K}, \operatorname{MoK}_{\alpha}(\lambda=0.71073 \AA), \theta_{\min }=1.93^{\circ}, \theta_{\max }=$ $26.15^{\circ}, 102298$ measured reflections $(-26 \leq h \leq 25,-19 \leq k$ $\leq 19,-26 \leq l \leq 26), 7303$ independent, 4923 observed reflections, $R_{\text {int }}=0.0967, R=0.0361, \mathrm{w} R=0.0881$, residual

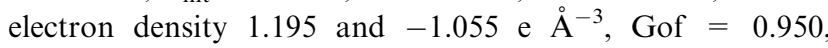
refinement program SHELXL-97, $N_{\text {ref }}=7303, N_{\text {par }}=376$.

\section{$\left\{\eta^{5}: \eta^{1}[2-(\right.$ tert-Butylphenylphosphanyl)ethyl]indenyl $\}$ - ( $\eta^{2}$-ethen)rhodium(I) (rac-18)}

At $-78{ }^{\circ} \mathrm{C}$ butyllithium in hexane $(2.3 \mathrm{~mL}, 3.7 \mathrm{mmol}, 1.6 \mathrm{M})$ was added dropwise to tert-butylphenylphosphane $(0.159 \mathrm{~g}$, $3.1 \mathrm{mmol})$ in THF $(15 \mathrm{~mL})$. After stirring for $2 \mathrm{~h}$ at $20^{\circ} \mathrm{C}$ [4.5]benzospiro[2.4]hepta-4,6-diene $(\mathbf{1 6}, 0.440 \mathrm{~g}, 3.1 \mathrm{mmol})$ was added. After stirring for $3 \mathrm{~d}$ at $65{ }^{\circ} \mathrm{C}$ the mixture was cooled to $-78{ }^{\circ} \mathrm{C}$, and $\left[\mathrm{Rh}_{2}\left(\mathrm{C}_{2} \mathrm{H}_{4}\right) \mathrm{Cl}_{2}\right](0.720 \mathrm{~g}, 1.9 \mathrm{mmol})$ was added, and the solution was warmed to $20{ }^{\circ} \mathrm{C}$. After $2 \mathrm{~h}$ the solvent was removed at reduced pressure, and the residue was taken up with diethyl ether $(5 \mathrm{~mL})$. After filtration through a frit covered with a $3 \mathrm{~cm}$ thick layer of Celite the solvent was removed at reduced pressure, and the residue was taken up with pentane $(5 \mathrm{~mL})$. Another filtration through a frit covered with a $3 \mathrm{~cm}$ thick layer of Celite afforded after solvent removal rhodium(I) chelate $\mathrm{rac}-\mathbf{1 8}$ [0.963 g, $2.2 \mathrm{mmol}, 71 \%$, diastereomeric mixture (10:1, ${ }^{1} \mathrm{H}$ NMR), purity $\geq 95 \%$ ( ${ }^{1} \mathrm{H}$ NMR)] as a brown oil.

IR (de 82\%): $\tilde{v}=3050\left(\mathrm{w}, \mathrm{C}_{2} \mathrm{H}_{4}\right), 2961(\mathrm{~m}, \mathrm{C}-\mathrm{H}), 2356(\mathrm{w})$, 2130 (w), 1948 (w), 1592 (w), 1430 (w, P-Ph), 1327 (w), 1260 (s, $\left.\mathrm{C}_{2} \mathrm{H}_{4}\right), 1124(\mathrm{~m}), 1091(\mathrm{~s}), 1017$ (s, $t$-Bu), $922(\mathrm{w}), 842(\mathrm{~m}, \mathrm{Cp})$, 797 (s, Cp), 731 (m), 697 (s) $\mathrm{cm}^{-1} .{ }^{1} \mathrm{H}-\mathrm{NMR}(400 \mathrm{MHz}$, $\mathrm{CDCl}_{3}$, major diastereomer): $\delta=0.74\left(\mathrm{~d},{ }^{3} J_{\mathrm{H}, \mathrm{P}}=13.3 \mathrm{~Hz}\right.$, $\left.9 \mathrm{H}, \mathrm{CH}_{3}\right), 2.11\left(\mathrm{~m}, 2 \mathrm{H}, \mathrm{PCH}_{2} \mathrm{CH}_{2}\right), 2.47\left(\mathrm{~d},{ }^{3} J_{\mathrm{H}, \mathrm{H}}=6.1 \mathrm{~Hz}\right.$, 
$\left.2 \mathrm{H},=\mathrm{CH}_{2}\right), 2.75\left(\mathrm{~m}, 2 \mathrm{H}, \mathrm{PCH}_{2}\right), 3.02\left(\mathrm{dt},{ }^{3} J_{\mathrm{H}, \mathrm{H}}=7.9\right.$, $\left.2.0 \mathrm{~Hz}, 2 \mathrm{H},=\mathrm{CH}_{2}\right), 5.36\left(\mathrm{~d},{ }^{3} J_{\mathrm{H}, \mathrm{H}}=2.9 \mathrm{~Hz}, 1 \mathrm{H}, \mathrm{PCH}_{2} \mathrm{CH}_{2^{-}}\right.$ $\mathrm{CCHCH}), 6.13\left(\mathrm{~d},{ }^{3} \mathrm{~J}_{\mathrm{H}, \mathrm{H}}=2.6 \mathrm{~Hz}, 1 \mathrm{H}, \mathrm{PCH}_{2} \mathrm{CH}_{2} \mathrm{CCH}\right), 6.41(\mathrm{~d}$, ${ }^{3} J_{\mathrm{H}, \mathrm{H}}=8.2 \mathrm{~Hz}, 1 \mathrm{H}, \mathrm{PCH}_{2} \mathrm{CH}_{2} \mathrm{CCCH}$ or $\left.\mathrm{PCH}_{2} \mathrm{CH}_{2} \mathrm{CCH}\right), 6.77$ (t, ${ }^{3} J_{\mathrm{H}, \mathrm{H}}=7.9 \mathrm{~Hz}, 1 \mathrm{H}, \mathrm{PCH}_{2} \mathrm{CH}_{2} \mathrm{CCCHCH}$ or $\mathrm{PCH}_{2} \mathrm{CH}_{2}-$ $\mathrm{CCHCH}), 6.93\left(\mathrm{~d},{ }^{3} J_{\mathrm{H}, \mathrm{H}}=8.2 \mathrm{~Hz}, 1 \mathrm{H}, \mathrm{PCH}_{2} \mathrm{CH}_{2} \mathrm{CCCH}\right.$ or $\mathrm{PCH}_{2} \mathrm{CH}_{2} \mathrm{CCH}$ ), $7.03\left(\mathrm{t},{ }^{3} \mathrm{~J}_{\mathrm{H}, \mathrm{H}}=7.2 \mathrm{~Hz}, 1 \mathrm{H}, \mathrm{PCH}_{2} \mathrm{CH}_{2^{-}}\right.$ $\mathrm{CCCHCH}$ or $\left.\mathrm{PCH}_{2} \mathrm{CH}_{2} \mathrm{CCHCH}\right), 7.19(\mathrm{~m}, 3 \mathrm{H}, o-, p-\mathrm{CH}), 7.52$ $(\mathrm{m}, 2 \mathrm{H}, m-\mathrm{H}) \mathrm{ppm} .{ }^{13} \mathrm{C}-\mathrm{NMR}\left(100.6 \mathrm{MHz}, \mathrm{CDCl}_{3}\right): \delta=19.3(\mathrm{~d}$, $\left.{ }^{2} J_{\mathrm{C}, \mathrm{P}}=2.4 \mathrm{~Hz}, \mathrm{PCH}_{2} \mathrm{CH}_{2}\right), 26.3\left(\mathrm{~d},{ }^{2} J_{\mathrm{C}, \mathrm{P}}=4.7 \mathrm{~Hz}, \mathrm{CH}_{3}\right)$, $32.0\left(\mathrm{~d},{ }^{1} J_{\mathrm{C}, \mathrm{P}}=16.7 \mathrm{~Hz},{ }^{2} J_{\mathrm{C}, \mathrm{Rh}}=3.7 \mathrm{~Hz}, \mathrm{CCH}_{3}\right), 35.2(\mathrm{dd}$, $\left.{ }^{2} J_{\mathrm{C}, \mathrm{P}}=13.9 \mathrm{~Hz},{ }^{1} J_{\mathrm{C}, \mathrm{Rh}}=1.3 \mathrm{~Hz},=\mathrm{CH}_{2}\right), 42.5\left(\mathrm{~d},{ }^{1} J_{\mathrm{C}, \mathrm{P}}=\right.$ $\left.23.7 \mathrm{~Hz}, \mathrm{PCH}_{2}\right), 77.0\left(\mathrm{dd},{ }^{5} J_{\mathrm{C}, \mathrm{P}}=13.2 \mathrm{~Hz},{ }^{1} J_{\mathrm{C}, \mathrm{Rh}}=2.6 \mathrm{~Hz}\right.$, $\left.\mathrm{PCH}_{2} \mathrm{CH}_{2} \mathrm{CCHCH}\right), 90.2\left(\mathrm{dd},{ }^{5} J_{\mathrm{C}, \mathrm{P}}=5.7 \mathrm{~Hz},{ }^{1} J_{\mathrm{C}, \mathrm{Rh}}=3.2 \mathrm{~Hz}\right.$, $\left.\mathrm{PCH}_{2} \mathrm{CH}_{2} \mathrm{CCH}\right), 102.6\left(\mathrm{dd},{ }^{4} J_{\mathrm{C}, \mathrm{P}}=6.3 \mathrm{~Hz},{ }^{1} J_{\mathrm{C}, \mathrm{Rh}}=\right.$ $4.7 \mathrm{~Hz}, \mathrm{PCH}_{2} \mathrm{CH}_{2} \mathrm{CC}$ or $\left.\mathrm{PCH}_{2} \mathrm{CH}_{2} \mathrm{CCC}\right), 109.2\left(\mathrm{~d},{ }^{3} J_{\mathrm{C}, \mathrm{P}}=\right.$ $\left.2.5 \mathrm{~Hz}, \mathrm{PCH}_{2} \mathrm{CH}_{2} \mathrm{C}\right), 113.6\left(\mathrm{~d},{ }^{4} J_{\mathrm{C}, \mathrm{P}}=2.1 \mathrm{~Hz}, \mathrm{PCH}_{2} \mathrm{CH}_{2} \mathrm{CC}\right.$ or $\left.\mathrm{PCH}_{2} \mathrm{CH}_{2} \mathrm{CCC}\right), 115.9\left(\mathrm{PCH}_{2} \mathrm{CH}_{2} \mathrm{CCCH}\right.$ or $\mathrm{PCH}_{2} \mathrm{CH}_{2}-$ $\mathrm{CCCCH})$, $118.0\left(\mathrm{PCH}_{2} \mathrm{CH}_{2} \mathrm{CCCH}\right.$ or $\left.\mathrm{PCH}_{2} \mathrm{CH}_{2} \mathrm{CCCCH}\right)$, $119.3\left(\mathrm{PCH}_{2} \mathrm{CH}_{2} \mathrm{CCCHCH}\right.$ or $\left.\mathrm{PCH}_{2} \mathrm{CH}_{2} \mathrm{CCCCHCH}\right), 123.4$ $\left(\mathrm{PCH}_{2} \mathrm{CH}_{2} \mathrm{CCCHCH}\right.$ or $\left.\mathrm{PCH}_{2} \mathrm{CH}_{2} \mathrm{CCCCHCH}\right), 127.7$ (d, $\left.{ }^{2} J_{\mathrm{C}, \mathrm{P}}=9.2 \mathrm{~Hz}, o-\mathrm{CH}\right), 129.5\left(\mathrm{~d},{ }^{4} J_{\mathrm{C}, \mathrm{P}}=2.1 \mathrm{~Hz}, p-\mathrm{CH}\right)$, $133.9\left(\mathrm{dd},{ }^{1} J_{\mathrm{C}, \mathrm{P}}=22.2 \mathrm{~Hz}, \mathrm{PCCH}\right), 134.1\left(\mathrm{~d},{ }^{3} J_{\mathrm{C}, \mathrm{P}}=11.3 \mathrm{~Hz}\right.$, $m$-CH) ppm. ${ }^{31} \mathrm{P}-\mathrm{NMR}\left(162 \mathrm{MHz}, \mathrm{C}_{6} \mathrm{D}_{6}\right): \delta=84.0\left(\mathrm{~d},{ }^{1} J_{\mathrm{P}, \mathrm{Rh}}\right.$ $=227.7 \mathrm{~Hz}) \mathrm{ppm}$. MS (de 82\%): $m / z(\%)=438(16)\left[\mathrm{M}^{+}\right]$, 410 (100) $\left[\mathrm{M}^{+}-\mathrm{CH}_{2}=\mathrm{CH}_{2}\right], 354$ (62), 231 (18), 197 (25), 135 (30), $57(12)\left[t-\mathrm{Bu}^{+}\right]$. HRMS $\left(\mathrm{M}^{+}=\mathrm{C}_{23} \mathrm{H}_{28} \mathrm{PRh}\right)$ calcd 438.0984, found 438.0987 .

\section{$\left\{\eta^{5}: \eta^{1}[2-(\right.$ tert-Butylphenylphosphanyl)ethyl]cyclopentadienyl $\}-$} $\left(\eta^{2}\right.$-cycloocten)iridium(I) (rac-19)

At $-78{ }^{\circ} \mathrm{C}$ butyllithium in hexane $(1.1 \mathrm{~mL}, 1.7 \mathrm{mmol}, 1.6 \mathrm{M})$ was added dropwise to tert-butylphenylphosphane $(0.269 \mathrm{~g}$, $1.6 \mathrm{mmol})$ in THF $(15 \mathrm{~mL})$. After stirring at $22{ }^{\circ} \mathrm{C}$ for $2 \mathrm{~h}$ spiro[2.4] hepta-4,6-diene $(4,0.15 \mathrm{~mL}, 1.6 \mathrm{mmol})$ was added, and the solution was stirred at $65^{\circ} \mathrm{C}$ for $2 \mathrm{~h}$. Then the mixture was cooled to $-78^{\circ} \mathrm{C}$, and $\left[\operatorname{Ir}(\mathrm{coe}){ }_{2} \mathrm{Cl}\right]_{2}(0.871 \mathrm{~g}, 1.0 \mathrm{mmol}$, coe $=$ cyclooctene) was added, and the mixture was slowly warmed to $20{ }^{\circ} \mathrm{C}$. After stirring for $12 \mathrm{~h}$ the solvent was removed at reduced pressure, and the residue was taken up with diethyl ether $(5 \mathrm{~mL})$. After filtration through a frit covered with a $3 \mathrm{~cm}$ thick layer of Celite $r a c-19(0.187 \mathrm{~g}$, $0.3 \mathrm{mmol}, 21 \%$ ) was isolated as a yellow oil. Residual cyclooctene could not be removed.

IR: $\tilde{v}=3055(\mathrm{w}), 2918(\mathrm{~s}, \mathrm{C}-\mathrm{H}), 2855(\mathrm{~m}, \mathrm{C}-\mathrm{H}), 2321(\mathrm{w})$, 2104 (w), 1947 (w), 1623 (m), 1463 (m, C-H), 1435 (s, P-Ph), $1392(\mathrm{w}), 1361(\mathrm{w}, t-\mathrm{Bu}), 1261(\mathrm{~m}), 1156(\mathrm{w}), 1100(\mathrm{~s}), 1015(\mathrm{~s}$, $t$-Bu), $920(\mathrm{w}), 805$ (s, Cp), $745(\mathrm{~s}, \mathrm{Cp}), 687(\mathrm{~s}) \mathrm{cm}^{-1} .{ }^{1} \mathrm{H}-\mathrm{NMR}$ $\left(400 \mathrm{MHz}, \mathrm{C}_{6} \mathrm{D}_{6}\right): \delta=0.93\left(\mathrm{~d},{ }^{2} J_{\mathrm{H}, \mathrm{P}}=13.0 \mathrm{~Hz}, 9 \mathrm{H}, \mathrm{CH}_{3}\right)$, 1.23-2.07 (m, 14H, coe- $\mathrm{H}), 2.64\left(\mathrm{~m}, 2 \mathrm{H}, \mathrm{PCH}_{2} \mathrm{CH}_{2}\right), 2.86(\mathrm{~m}$, $\left.2 \mathrm{H}, \mathrm{PCH}_{2}\right), 4.70\left(\mathrm{~d}, J_{\mathrm{H}, \mathrm{P}}=2.4 \mathrm{~Hz}, 1 \mathrm{H}, \mathrm{PCH}_{2} \mathrm{CH}_{2} \mathrm{CCHCH}\right)$, $4.86\left(\mathrm{~s}, 1 \mathrm{H}, \mathrm{PCH}_{2} \mathrm{CH}_{2} \mathrm{CCH}\right), 5.02\left(\mathrm{~s}, 1 \mathrm{H}, \mathrm{PCH}_{2} \mathrm{CH}_{2} \mathrm{CCHCH}\right)$, $5.28\left(\mathrm{~s}, 1 \mathrm{H}, \mathrm{PCH}_{2} \mathrm{CH}_{2} \mathrm{CCH}\right), 7.14(\mathrm{~m}, 3 \mathrm{H}, o-, p-\mathrm{H}), 7.93(\mathrm{t}$, $\left.{ }^{3} J_{\mathrm{H}, \mathrm{P}}=8.2 \mathrm{~Hz}, 2 \mathrm{H}, m-\mathrm{H}\right) \mathrm{ppm} .{ }^{13} \mathrm{C}-\mathrm{NMR}(100.6 \mathrm{MHz}$, $\left.\mathrm{C}_{6} \mathrm{D}_{6}\right): \delta=21.6\left(\mathrm{~d},{ }^{2} J_{\mathrm{C}, \mathrm{P}}=0.7 \mathrm{~Hz}, \mathrm{PCH}_{2} \mathrm{CH}_{2}\right), 27.0(\mathrm{~d}$, $\left.{ }^{2} J_{\mathrm{C}, \mathrm{P}}=4.0 \mathrm{~Hz}, \mathrm{CH}_{3}\right), 31.6\left(\mathrm{~d},{ }^{1} J_{\mathrm{C}, \mathrm{P}}=23.9 \mathrm{~Hz}, \mathrm{CCH}_{3}\right), 33.2$ (coe-C), 34.8 (coe-C), 36.6 (coe-C), 36.8 (coe-C), 46.4 (d, $\left.{ }^{1} J_{\mathrm{C}, \mathrm{P}}=46.5 \mathrm{~Hz}, \mathrm{PCH}_{2}\right), 72.0\left(\mathrm{~d}, J_{\mathrm{C}, \mathrm{P}}=1.9 \mathrm{~Hz}, \mathrm{PCH}_{2} \mathrm{CH}_{2^{-}}\right.$
$\mathrm{CCHCH}), 76.8\left(\mathrm{~d}, J_{\mathrm{C}, \mathrm{P}}=2.4 \mathrm{~Hz}, \mathrm{PCH}_{2} \mathrm{CH}_{2} \mathrm{CCHCH}\right), 83.7$ $\left(\mathrm{d}, J_{\mathrm{C}, \mathrm{P}}=9.5 \mathrm{~Hz}, \mathrm{PCH}_{2} \mathrm{CH}_{2} \mathrm{CCH}\right), 89.8\left(\mathrm{~d}, J_{\mathrm{C}, \mathrm{P}}=8.2 \mathrm{~Hz}\right.$, $\left.\mathrm{PCH}_{2} \mathrm{CH}_{2} \mathrm{CCH}\right), 105.1\left(\mathrm{~d},{ }^{3} J_{\mathrm{C}, \mathrm{P}}=5.9 \mathrm{~Hz}, \mathrm{PCH}_{2} \mathrm{CH}_{2} \mathrm{C}\right), 127.4$ $\left(\mathrm{d},{ }^{2} J_{\mathrm{C}, \mathrm{P}}=9.0 \mathrm{~Hz}, o-\mathrm{CH}\right), 129.3\left(\mathrm{~d},{ }^{4} J_{\mathrm{C}, \mathrm{P}}=2.3 \mathrm{~Hz}, p-\mathrm{CH}\right)$, $134.4\left(\mathrm{~d},{ }^{3} J_{\mathrm{C}, \mathrm{P}}=9.7 \mathrm{~Hz}, m-\mathrm{CH}\right), 136.5\left(\mathrm{~d},{ }^{1} J_{\mathrm{C}, \mathrm{P}}=34.1 \mathrm{~Hz}\right.$, $\mathrm{PCCH})$ ppm. ${ }^{31} \mathrm{P}$ NMR $\left(162 \mathrm{MHz}, \mathrm{C}_{6} \mathrm{D}_{6}\right): \delta=35.3 \mathrm{ppm}$. MS: $m / z(\%)=560(12)\left[\mathrm{M}^{+}\right], 450(12)\left[\mathrm{M}^{+}-\right.$coe], $392(47), 366$ (100), 257 (19) $\left[\mathrm{M}^{+}-\mathrm{Ir}-\right.$ coe]. HRMS $\left(\mathrm{M}^{+}=\mathrm{C}_{25} \mathrm{H}_{36} \mathrm{IrP}\right)$ calcd 560.2184, found 560.2180 .

\section{Chloro- $\left\{\eta^{5}: \eta^{1}[2-(\right.$ tert-butylphenylphosphanyl)ethyl]- cyclopentadienyl\}-nickel(II) (rac-20)}

At $-78^{\circ} \mathrm{C}$ butyllithium in hexane $(6.4 \mathrm{~mL}, 10.2 \mathrm{mmol}, 1.6 \mathrm{M})$ was added dropwise to tert-butylphenylphosphane $(1.438 \mathrm{~g}$, $8.7 \mathrm{mmol})$ in THF $(30 \mathrm{~mL})$. After stirring at $20{ }^{\circ} \mathrm{C}$ for $2 \mathrm{~h}$ spiro[2.4]hepta-4,6-diene $(\mathbf{4}, 0.9 \mathrm{~mL}, 9.0 \mathrm{mmol})$ was added, and the solution was stirred at $65^{\circ} \mathrm{C}$ for $2 \mathrm{~h}$. Then the mixture was cooled to $-78{ }^{\circ} \mathrm{C}$, and $\mathrm{NiCl}_{2}(1.350 \mathrm{~g}, 10.3 \mathrm{mmol})$ was added, and the mixture was slowly warmed to $20{ }^{\circ} \mathrm{C}$. After stirring for $1 \mathrm{~h}$ the solvent was removed at reduced pressure, and the residue was taken up with diethyl ether $(5 \mathrm{~mL})$. After filtration through a frit covered with a $3 \mathrm{~cm}$ thick layer of Celite, solvent removal at reduced pressure, and purification of the residue by column chromatography $\left(3 \times 20 \mathrm{~cm}, \mathrm{SiO}_{2}\right.$, TBME) rac-20 $[2.122 \mathrm{~g}, 6.1 \mathrm{mmol}, 70 \%$, purity $\geq 95 \%$ $\left.\left({ }^{1} \mathrm{H}-\mathrm{NMR}\right)\right]$ was isolated as a purple solid, $\mathrm{mp} 105.5{ }^{\circ} \mathrm{C}$.

IR: $\tilde{v}=3077(\mathrm{w}), 2945(\mathrm{~m}, \mathrm{C}-\mathrm{H}), 2916(\mathrm{~m}), 2862(\mathrm{~m}, \mathrm{C}-\mathrm{H})$, 1610 (w), 1460 (m, C-H), 1437 (m, P-Ph), 1394 (w), 1361 (m, $t$-Bu), $1183(\mathrm{~m}), 1162(\mathrm{~m}), 1102(\mathrm{~m}), 1022(\mathrm{~m}, t-\mathrm{Bu}), 847(\mathrm{~m}$, Cp), 785 (s, Cp), 755 (s), $688(\mathrm{~s}), 617(\mathrm{~m}) \mathrm{cm}^{-1} \cdot{ }^{1} \mathrm{H}-\mathrm{NMR}$ $\left(400 \mathrm{MHz}, \mathrm{CDCl}_{3}\right): \delta=1.43\left(\mathrm{~d},{ }^{3} J_{\mathrm{H}, \mathrm{P}}=15.1 \mathrm{~Hz}, 9 \mathrm{H}, \mathrm{CH}_{3}\right)$, $1.39\left(\mathrm{~m}, 1 \mathrm{H}, \mathrm{PCH}_{2} \mathrm{CH}_{2}\right), 1.45\left(\mathrm{~m}, 2 \mathrm{H}, \mathrm{PCH}_{2} \mathrm{CH}_{2}\right), 2.44(\mathrm{~m}$, $\left.1 \mathrm{H}, \mathrm{PCH}_{2}\right), 2.69\left(\mathrm{~m}, 1 \mathrm{H}, \mathrm{PCH}_{2}\right), 5.55\left(\mathrm{dd},{ }^{3} J_{\mathrm{H}, \mathrm{P}}=1.3\right.$, $\left.2.2 \mathrm{~Hz}, 1 \mathrm{H}, \mathrm{PCH}_{2} \mathrm{CH}_{2} \mathrm{CCH}\right), 5.76\left(\mathrm{~d},{ }^{3} J_{\mathrm{H}, \mathrm{P}}=2.1 \mathrm{~Hz}, 1 \mathrm{H}\right.$, $\left.\mathrm{PCH}_{2} \mathrm{CH}_{2} \mathrm{CCH}\right), 5.82\left(\mathrm{dt},{ }^{3} J_{\mathrm{H}, \mathrm{P}}=1.5,3.3 \mathrm{~Hz}, 1 \mathrm{H}, \mathrm{PCH}_{2} \mathrm{CH}_{2}-\right.$ $\mathrm{CCHCH}), 5.96\left(\mathrm{t},{ }^{3} \mathrm{~J}_{\mathrm{H}, \mathrm{P}}=1.7 \mathrm{~Hz}, 1 \mathrm{H}, \mathrm{PCH}_{2} \mathrm{CH}_{2} \mathrm{CCHCH}\right)$, $7.62\left(\mathrm{t},{ }^{2} J_{\mathrm{H}, \mathrm{P}}=2.6 \mathrm{~Hz}, 3 \mathrm{H}, o-, p-\mathrm{H}\right), 8.30\left(\mathrm{ddd},{ }^{3} J_{\mathrm{H}, \mathrm{P}}=1.6\right.$, 7.9, $10.1 \mathrm{~Hz}, 2 \mathrm{H}, m-\mathrm{H}) \mathrm{ppm} .{ }^{13} \mathrm{C}-\mathrm{NMR}\left(100.6 \mathrm{MHz}, \mathrm{CDCl}_{3}\right)$ : $\delta=22.2\left(\mathrm{~d},{ }^{2} J_{\mathrm{C}, \mathrm{P}}=4.4 \mathrm{~Hz}, \mathrm{PCH}_{2} \mathrm{CH}_{2}\right), 25.5\left(\mathrm{~d},{ }^{2} J_{\mathrm{C}, \mathrm{P}}=3.8\right.$, $\left.\mathrm{CH}_{3}\right), 30.7\left(\mathrm{~d},{ }^{1} J_{\mathrm{C}, \mathrm{P}}=21.2 \mathrm{~Hz}, \mathrm{PCCH} \mathrm{CH}_{3}\right), 33.4\left(\mathrm{~d},{ }^{1} J_{\mathrm{C}, \mathrm{P}}=27.0\right.$, $\left.\mathrm{PCH}_{2}\right), 93.7\left(\mathrm{PCH}_{2} \mathrm{CH}_{2} \mathrm{CCHCH}\right), 94.6\left(\mathrm{~d},{ }^{3} J_{\mathrm{C}, \mathrm{P}}=7.0 \mathrm{~Hz}\right.$, $\left.\mathrm{PCH}_{2} \mathrm{CH}_{2} \mathrm{C}\right), 96.6\left(\mathrm{~d},{ }^{4} J_{\mathrm{C}, \mathrm{P}}=6.3 \mathrm{~Hz}, \mathrm{PCH}_{2} \mathrm{CH}_{2} \mathrm{CCH}\right)$, $98.1\left(\mathrm{PCH}_{2} \mathrm{CH}_{2} \mathrm{CCHCH}\right), 99.6\left(\mathrm{~d},{ }^{4} J_{\mathrm{C}, \mathrm{P}}=5.5 \mathrm{~Hz}\right.$, $\left.\mathrm{PCH}_{2} \mathrm{CH}_{2} \mathrm{CCH}\right), 127.4\left(\mathrm{~d},{ }^{2} J_{\mathrm{C}, \mathrm{P}}=9.3 \mathrm{~Hz}, o-\mathrm{CH}\right), 127.8(\mathrm{~d}$, $\left.{ }^{1} J_{\mathrm{C}, \mathrm{P}}=32.5, \mathrm{PCCH}\right), 130.0\left(\mathrm{~d},{ }^{4} J_{\mathrm{C}, \mathrm{P}}=2.3 \mathrm{~Hz}, p-\mathrm{CH}\right), 132.6$ $\left(\mathrm{d},{ }^{3} J_{\mathrm{C}, \mathrm{P}}=10.1 \mathrm{~Hz}, m-\mathrm{CH}\right) \mathrm{ppm} .{ }^{31} \mathrm{P}-\mathrm{NMR}(162 \mathrm{MHz}$, $\left.\mathrm{CDCl}_{3}\right): \delta=99.3$ ppm. MS: $m / z(\%)=350(76)\left[\mathrm{M}^{+}\right], 358$ (100) $\left[\mathrm{M}^{+}-\mathrm{NiCl}\right], 182(50), 126(59), 91$ (11), $79(10), 57$ (13) $\left[t-\mathrm{Bu}^{+}\right]$. HRMS $\left(\mathrm{M}^{+}=\mathrm{C}_{17} \mathrm{H}_{22} \mathrm{NiPCl}\right)$ calcd 350.0501 , found 350.0499 .

\section{Dichloro $\left\{\eta^{5}: \eta^{1}[2\right.$-(tert-butylphenylphosphanyl)ethyl]- cyclopentadienyl\}-chromium(III) (rac-21)}

At $-78{ }^{\circ} \mathrm{C}$ butyllithium in hexane $(2.9 \mathrm{~mL}, 3.7 \mathrm{mmol}, 1.6 \mathrm{M})$ was added dropwise to tert-butylphenylphosphane $(0.518 \mathrm{~g}$, $3,1 \mathrm{mmol})$ in THF $(15 \mathrm{~mL})$. After stirring at $20{ }^{\circ} \mathrm{C}$ for $2 \mathrm{~h}$ spiro[2.4]hepta-4,6-diene $(\mathbf{4}, 0.3 \mathrm{~mL}, 3.3 \mathrm{mmol})$ was added, and the solution was stirred at $65^{\circ} \mathrm{C}$ for $2 \mathrm{~h}$. Then the mixture 
was cooled to $-78{ }^{\circ} \mathrm{C}$, and $\mathrm{Cr}(\mathrm{THF})_{3} \mathrm{Cl}_{3}(1.16 \mathrm{~g}, 3.1 \mathrm{mmol})$ was added, and the mixture was slowly warmed to $20^{\circ} \mathrm{C}$. After stirring for $12 \mathrm{~h}$ the solvent was removed at reduced pressure, and the residue was washed with pentane and then taken up with boiling toluene $(10 \mathrm{~mL})$. After filtration through a frit covered with a $3 \mathrm{~cm}$ thick layer of Celite, the solution was concentrated and cooled to $-25^{\circ} \mathrm{C}$. rac $-21(0.570 \mathrm{~g}, 1.5 \mathrm{mmol}$, $48 \%$ ) was obtained as a blue solid, $\mathrm{mp} 239.9^{\circ} \mathrm{C}$.

IR: $\tilde{v}=3092(\mathrm{w}), 3055(\mathrm{w}), 2959(\mathrm{w}, \mathrm{C}-\mathrm{H}), 2361(\mathrm{w}), 1474$ (m, C-H), 1435 (m, P-Ph), 1366 (m, $t$-Bu), $1172(\mathrm{~m}), 1100(\mathrm{~m})$, 1053 (w), 892 (w), 819 (m), 810 (s, Cp), 747 (s, Cp), 699 (s) cm $\mathrm{cm}^{-1}$. MS: $m / z(\%)=379(30)\left[\mathrm{M}^{+}\right], 288(54), 286(100), 202(44)$, 124 (26), 109 (37), 91 (36), 57 (76) $\left[t-\mathrm{Bu}^{+}\right]$. HRMS $\left(\mathrm{M}^{+}=\right.$ $\mathrm{C}_{17} \mathrm{H}_{22} \mathrm{Cl}_{2} \mathrm{CrP}$ ) calcd 379.0241, found 379.0242. Anal. $\left(\mathrm{C}_{17} \mathrm{H}_{22} \mathrm{Cl}_{2} \mathrm{CrP}\right)$ calcd C 52.86; H 7.30, found C 52.90; H 7.03.

Crystal structure analysis: ${ }^{45}$ empirical formula $\mathrm{C}_{17} \mathrm{H}_{22} \mathrm{Cl}_{2} \mathrm{CrP}$, molecular weight 380.22 , crystal system monoclinic, space group $P 2_{1} / c, a=15.428(3) \AA, b=16.376(5) \AA, c=$ $15.847(3) \AA, \alpha=90.00^{\circ}, \beta=115.182(19)^{\circ}, \gamma=90.00^{\circ}$, $V=3623.3(14) \AA^{3}, Z=8, d_{\text {calcd }}=1.394 \mathrm{~g} \mathrm{~cm}^{-1}$, $F(000)=1576.0, \mu=1.006 \mathrm{~mm}^{-1}$, Stoe IPDS diffractometer, $T=297 \mathrm{~K}, \operatorname{MoK}_{\alpha}(\lambda=0.71073 \AA), \theta_{\min }=1.92^{\circ}, \theta_{\max }=$ $26.14^{\circ}, 50517$ measured reflections $(-18 \leq h \leq 18,-20 \leq$ $k \leq 20,-19 \leq l \leq 19), 7080$ independent, 3591 observed reflections, $R_{\text {int }}=0.1203, R=0.0343, \mathrm{w} R=0.0558$, residual electron density 0.366 and $-0.237 \mathrm{e} \AA^{-3}$, Gof $=0.841$, refinement program SHELXL-97, $N_{\text {ref }}=3591, N_{\text {par }}=379$.

\section{Acknowledgements}

We thank Dr Michael Wiebcke for his help with the crystal structure analyses.

\section{References}

1 J. Hartwig, Organotransition Metal Chemistry, University Science Books, Sausalito, 2010.

2 Transition Metals for Organic Synthesis, ed. M. Beller and C. Bolm, Wiley-VCH, Weinheim, 1998.

3 Ferrocenes: Ligands, Materials and Biomolecules, ed. P. Stepnicka, John Wiley \& Sons, Chichester, 2008.

4 Carbon-Rich Compounds: From Molecules to Materials, ed. M. M. Haley and R. R. Tykwinski, Wiley-VCH, Weinheim, 2006.

5 F. Le Bideau, M. Salmain, S. Top and G. Jaouen, Chem.-Eur. J., 2001, 7, 2289-2294.

6 F. Le Bideau, E. B. Kaloum, P. Haquette, U. Kernbach, E. Stephan, S. Top, A. Vessieres, G. Jaouen and J. Marrot, Chem. Commun., 2000, 211-212.

7 I. Baumgardt and H. Butenschön, Eur. J. Org. Chem., 2010, 1076-1087.

8 J. Ma, M. Vollmann, H. Menzel, S. Pohle and H. Butenschön, J. Inorg. Organomet. Polym. Mater., 2008, 18, 41-50.

9 M. Vollmann and H. Butenschön, C. R. Chim., 2005, 8, 1282-1285.

10 M. Wagner, Angew. Chem., 2006, 118, 6060-6062 (Angew. Chem., Int. Ed., 2006, 45, 5916-5918).

11 U. H. F. Bunz, J. Organomet. Chem., 2003, 683, 269-287.

12 R. K. Bohn and A. Haaland, J. Organomet. Chem., 1966, 5, 470-476.

13 H. Butenschön, Chem. Rev., 2000, 100, 1527-1564.

14 U. Siemeling, Chem. Rev., 2000, 100, 1495-1526.

15 M. Hussain, S. Kohser, K. Janssen, R. Wartchow and H. Butenschön, Organometallics, 2009, 28, 5212-5221.

16 M. Hussain, D. Albert, R. Wartchow and H. Butenschön, Chem.-Asian J., 2007, 2, 782-793.

17 S. I. Kozhushkov, J. Foerstner, A. Kakoschke, D. Stellfeldt, L. Yong, R. Warchow, A. de Meijere and H. Butenschön, Chem.-Eur. J., 2006, 12, 5642-5647.
18 L. Yong, E. Hofer, R. Wartchow and H. Butenschön, Organometallics, 2003, 22, 5463-5467.

19 A. Kakoschke, L. Yong, R. Wartchow and H. Butenschön, J. Organomet. Chem., 2003, 674, 86-95.

20 L. Yong and H. Butenschön, Chem. Commun., 2002, 2852-2853.

21 J. Foerstner, A. Kakoschke, R. Goddard, J. Rust, R. Wartchow and H. Butenschön, J. Organomet. Chem., 2001, 617/618, 412-422.

22 J. Foerstner, A. Kakoschke, R. Wartchow and H. Butenschön, Organometallics, 2000, 19, 2108-2113.

23 J. Foerstner, R. Wartchow and H. Butenschön, New J. Chem., 1998, 22, 1155-1157.

24 J. Foerstner, S. Kozhushkov, P. Binger, P. Wedemann, M. Noltemeyer, A. de Meijere and H. Butenschön, Chem. Commun., 1998, 239-240.

25 J. Foerstner, A. Kakoschke, D. Stellfeldt, H. Butenschön and R. Wartchow, Organometallics, 1998, 17, 893-896.

26 J. Foerstner, F. Olbrich and H. Butenschön, Angew. Chem., 1996, 108, 1323-1325 (Angew. Chem., Int. Ed. Engl., 1996, 35, 1234-1237).

27 J. Foerstner, R. Kettenbach, R. Goddard and H. Butenschön, Chem. Ber., 1996, 129, 319-325.

28 R. T. Kettenbach, W. Bonrath and H. Butenschön, Chem. Ber., 1993, 126, 1657-1669.

29 H. Butenschön, R. T. Kettenbach and C. Krüger, Angew. Chem., 1992, 104, 1052-1054 (Angew. Chem., Int. Ed. Engl., 1992, 31, 1066-1068).

30 R. T. Kettenbach and H. Butenschön, New J. Chem., 1990, 14, 599-601.

31 T. Ishiyama, T. Mizuta, K. Miyoshi and H. Nakazawa, Organometallics, 2003, 22, 1096-1105.

32 T. Ishiyama, H. Nakazawa and K. Miyoshi, J. Organomet. Chem., $2002,648,231-236$

33 T. Ishiyama, T. Mizuta, K. Miyoshi and H. Nakazawa, Chem. Lett., 2003, 70-71.

34 T. Koch, S. Blaurock, F. B. Somoza Jr., A. Voigt, R. Kirmse and E. Hey-Hawkins, Organometallics, 2000, 19, 2556-2563.

35 C. Kaulen, C. Pala, C. Hu and C. Ganter, Organometallics, 2001, 20, 1614-1619.

36 L. Jekki, C. Pala, B. Calmuschi and C. Ganter, Eur. J. Inorg. Chem., 2005, 745-750.

37 J. Bitta, S. Fassbender, G. Reiss, W. Frank and C. Ganter, Organometallics, 2005, 24, 5176-5179.

38 M. Nieuwenhuyzen, G. C. Saunders and E. C. M. S. Smyth, Organometallics, 2006, 25, 996-1003.

39 R. M. Bellabarba, M. Nieuwenhuyzen and G. C. Saunders, Organometallics, 2003, 22, 1802-1810.

40 M. Nieuwenhuyzen and G. C. Saunders, J. Organomet. Chem., 2000, 595, 292-295.

41 R. M. Bellabarba, M. Nieuwenhuyzen and G. C. Saunders, Organometallics, 2002, 21, 5726-5737.

42 T. Kauffmann, J. Ennen, H. Lhotak, A. Rensing, F. Steinseifer and A. Woltermann, Angew. Chem., 1980, 92, 321-323 (Angew. Chem., Int. Ed. Engl., 1980, 19, 328-329).

43 M. Hesse, H. Meier and B. Zeeh, Spektroskopische Methoden in der organischen Chemie, Thieme, Stuttgart, 2005.

44 R. Benn, Org. Magn. Reson., 1983, 21, 723-726.

$45 \mathrm{CCDC} 820401$ (rac-6), 820403 (rac-14), and 820402 (rac-21) contain the supplementary crystallographic data for this paper.

46 V. Gandon, N. Agenet, K. P. C. Vollhardt, M. Malacria and C. Aubert, J. Am. Chem. Soc., 2009, 131, 3007-3015.

47 A. H. Janowicz, H. E. Bryndza and R. G. Bergman, J. Am. Chem. Soc., 1981, 103, 1516-1518.

48 M. Tasi, T. Ranga and G. Pályi, in Organometallic Syntheses, ed. R. B. King and J. J. Eisch, Elsevier, New York, 1988, pp. 262-265.

49 R. T. Kettenbach, W. Bonrath and H. Butenschön, Chem. Ber., 1993, 126, 1657-1669.

50 I. Lee, F. Dahan, A. Maisonnat and R. Poilblanc, Organometallics, 1994, 13, 2743-2750.

51 Y. Kataoka, Y. Saito, K. Nagata, K. Kitamura, A. Shibahara and K. Tani, Chem. Lett., 1995, 833-834.

52 Y. Kataoka, Y. Saito, K. Nagata, K. Kitamura, A. Shibahara and K. Tani, Chem. Lett., 1996, 577.

53 Y. Kataoka, Y. Saito, A. Shibahara and K. Tani, Chem. Lett., $1997,621-622$.

54 Y. Kataoka, Y. Nakagawa, A. Shibahara, T. Yamagata, K. Mashima and K. Tani, Organometallics, 2004, 23, 2095-2099. 
55 Y. Kataoka, Y. Iwato, T. Yamagata and K. Tani, Organometallics, 1999, 18, 5423-5425.

56 Y. Kataoka, Y. Iwato, A. Shibahara, T. Yamagata and K. Tani, Chem. Commun., 2000, 841-842.

57 L. P. Barthel-Rosa, V. J. Catalano and J. H. Nelson, J. Chem. Soc., Chem. Commun., 1995, 1629-1630.

58 L. P. Barthel-Rosa, V. J. Catalano, K. Maitra and J. H. Nelson, Organometallics, 1996, 15, 3924-3934.

59 M. J. Atherton, J. Fawcett, J. H. Holloway, E. G. Hope, A. Karaçar, D. R. Russell and G. C. Saunders, J. Chem. Soc., Dalton Trans., 1996, 3215-3220.

60 M. J. Atherton, J. Fawcett, J. H. Holloway, E. G. Hope, A. Karaçar, D. R. Russell and G. C. Saunders, J. Chem. Soc., Chem. Commun., 1995, 191-192.

61 J. Fawcett, S. Friedrichs, J. H. Holloway, E. G. Hope, V. McKee, M. Nieuwenhuyzen, D. R. Russel and G. C. Saunders, J. Chem. Soc., Dalton Trans., 1998, 1477-1484.

62 Y. Nishibayashi, I. Takei and M. Hidai, Organometallics, 1997, 16, 3091-3093.

63 L. Lefort, T. W. Crane, M. D. Farwell, D. M. Baruch, J. A. Kaeuper, R. J. Lachicotte and W. D. Jones, Organometallics, 1998, 17, 3889-3899.

64 R. M. Bellabarba, G. P. Clancy, P. T. Gomes, A. M. Martins, L. H. Rees and M. L. H. Green, J. Organomet. Chem., 2001, 640, 93-112.

65 A. Doppiu, U. Englert and A. Salzer, Chem. Commun., 2004, 2166-2167.
66 S. Ciruelos, U. Englert, A. Salzer, C. Bolm and A. Maischak, Organometallics, 2000, 19, 2240-2242.

67 S. Ciruelos, A. Doppiu, U. Englert and A. Salzer, J. Organomet. Chem., 2002, 663, 183-191.

68 C. C. Brasse, U. Englert, A. Salzer, H. Waffenschmidt and P. Wasserscheid, Organometallics, 2000, 19, 3818-3823.

69 A. C. McConnell, J. P. Pogorzelec, A. M. Z. Slawin, L. G. Williams, I. P. P. Elliott, A. Haynes, A. C. Marr and J. D. Cole-Hamilton, J. Chem. Soc., Dalton Trans., 2006, 91-107.

70 M. Bernechea, J. R. Berenguer, E. Lalinde and J. Torroba, Organometallics, 2009, 28, 312-320.

71 D. C. Brookings, S. A. Harrison, R. J. Whitby, B. Crombie and R. V. H. Jones, Organometallics, 2001, 20, 4574-4583.

72 Y. Kataoka, A. Shibahara, Y. Saito, T. Yamagata and K. Tani, Organometallics, 1998, 17, 4338-4340.

73 Y. Kataoka, A. Shibahara, T. Yamagata and K. Tani, Organometallics, 2001, 20, 2431-2433.

74 A. Doppiu, U. Englert, V. Peters and A. Salzer, J. Organomet. Chem., 2007, 692, 4495-4505.

75 A. Doppiu, U. Englert, V. Peters and A. Salzer, Inorg. Chim. Acta, 2004, 357, 1773-1780.

76 A. Döhring, V. R. Jensen, P. W. Jolly, W. Thiel and J. C. Weber, Organometallics, 2001, 20, 2234-2245.

77 C. F. Wilcox Jr. and R. R. Craig, J. Am. Chem. Soc., 1961, 83, 3866-3871.

78 W. C. Still, M. Kahn and A. Mitra, J. Org. Chem., 1978, 43, 2923-2925. 